\title{
Approximate Controllability of Semilinear Neutral Stochastic Integrodifferential Inclusions with Infinite Delay
}

\author{
Meili Li and Man Liu \\ School of Science, Donghua University, Shanghai 201620, China \\ Correspondence should be addressed to Meili Li; stylml@dhu.edu.cn
}

Received 29 July 2015; Revised 23 October 2015; Accepted 28 October 2015

Academic Editor: Chris Goodrich

Copyright ( $) 2015$ M. Li and M. Liu. This is an open access article distributed under the Creative Commons Attribution License, which permits unrestricted use, distribution, and reproduction in any medium, provided the original work is properly cited.

\begin{abstract}
The approximate controllability of semilinear neutral stochastic integrodifferential inclusions with infinite delay in an abstract space is studied. Sufficient conditions are established for the approximate controllability. The results are obtained by using the theory of analytic resolvent operator, the fractional power theory, and the theorem of nonlinear alternative for Kakutani maps. Finally, an example is provided to illustrate the theory.
\end{abstract}

\section{Introduction}

Controllability is an important concept which plays a vital role in many areas of applied mathematics. For the last decades, many authors established sufficient conditions for the controllability of nonlinear systems in Banach spaces; see [1-3]. Most of the controllability results for nonlinear systems concern the so-called semilinear control systems that consist of a linear part and a nonlinear part. Chang et al. [4] investigated the controllability of semilinear differential systems with nonlocal initial conditions in Banach spaces.

Integrodifferential equations have been recently proved to be strong tools in the modeling of many phenomena arising from many fields such as electronics. The theory of integrodifferential inclusions in deterministic cases may be found in several papers and monographs; for example, see [5-8]. In fact, many deterministic models often fluctuate because of noise, so we must move from deterministic control problems to stochastic control problems. The applications of stochastic differential equations and stochastic delay differential equations have attracted great interest; see [9-13]. In particular, Balasubramaniam and Ntouyas [14] have studied the controllability of the following neutral stochastic functional differential inclusions with infinite delay in abstract space:

$$
\begin{aligned}
d\left[x(t)-f\left(t, x_{t}\right)\right] \in[ & A x(t)+B u(t)] d t \\
& +G\left(t, x_{t}\right) d \omega(t), \quad t \in[0, b],
\end{aligned}
$$

$$
\begin{aligned}
& x(t)=\phi(t) \in L_{2}(\Omega, \mathscr{B}), \\
& \text { for a.e } t \in(-\infty, 0],
\end{aligned}
$$

where $f:[0, b] \times \mathscr{B} \rightarrow H$ and $G:[0, b] \times \mathscr{B} \rightarrow$ $\mathscr{P}\left(L_{\mathrm{Q}}(K, H)\right)$ are, respectively, measurable and multivalued measurable mapping. They achieved controllability for (1) by assuming the semigroup $T(t)$ is compact and uniformly bounded and applying the theory of fractional power operators.

The concept of complete controllability is usually too strong which has limited applicability. Compared with complete controllability, approximate controllability is a weaker concept and it is completely adequate in applications; see [15-17]. Recently, Mokkedem and Fu [18] have studied the approximate controllability of the following semilinear neutral integrodifferential systems with finite delay:

$$
\begin{aligned}
\frac{d}{d t}\left[x(t)+f\left(t, x_{t}\right)\right]= & -A x(t)+\int_{0}^{t} \gamma(t-s) x(s) d s \\
& +G\left(t, x_{t}\right)+B u(t), \\
& t \in[0, T], \\
x_{0}=\phi, \quad t \in[-r, 0], &
\end{aligned}
$$


where $f:[0, T] \times C_{\alpha} \rightarrow H_{\alpha+\beta}$ and $G:[0, T] \times C_{\alpha} \rightarrow H$. They studied the problem by applying the theory of fractional power operators and $\alpha$-norm. Meanwhile, they supposed that the operator $(-A, D(-A))$ generates a compact analytic semigroup on $H$ and the corresponding linear system of (2) is approximately controllable. They established sufficient conditions of approximate controllability for the neutral integrodifferential control system in Hilbert space under a resolvent condition; in particular, they did not require that the resolvent operator $W(t)$ is compact for $t>0$.

In fact, neutral stochastic integrodifferential inclusions arise in many areas for applied mathematics. The approximate controllability for neutral stochastic integrodifferential inclusions is also an important and interesting topic for mathematics, but it is also a difficult problem. Meanwhile, few researches have been done on the approximate controllability for semilinear neutral stochastic integrodifferential inclusions with infinite delay. Motivated by [14, 18], we will show the approximate controllability of the following semilinear neutral stochastic integrodifferential inclusions with infinite delay in a Hilbert space:

$$
\begin{aligned}
& d\left[x(t)+F\left(t, x_{t}\right)\right] \\
& \in\left[-A x(t)+B u(t)+\int_{0}^{t} \gamma(t-s) x(s) d s\right] d t \\
& \quad+G\left(t, x_{t}\right) d \omega(t), \quad t \in J:=[0, T], \\
& x(t)=\phi(t) \in L_{2}\left(\Omega, \mathscr{B}_{\alpha}\right),
\end{aligned}
$$

$$
\text { for a.e } t \in J_{0}:=(-\infty, 0] \text {, }
$$

where $-A$ is the infinitesimal generator of an analytic semigroup of bound linear operator $S(t)_{t \geq 0}$, on a separable Hilbert space $H$ with inner product $\langle\cdot, \cdot\rangle$ and norm $\|\cdot\|$. The control function $u(\cdot)$ takes values in $L_{2}(J, U)$ of admissible control functions for a separable Hilbert space $U$ and $B$ is a bounded linear operator from $U$ into $H . \gamma(\cdot)$ is a family of closed linear operators to be specified later. Let $K$ be another separable Hilbert space with inner product $\langle\cdot, \cdot\rangle_{K}$ and norm $\|\cdot\|_{K}$. Suppose $\{\omega(t)\}_{t \geq 0}$ is a given $K$-valued Wiener process in a finite trace nuclear covariance operator $Q \geq 0 . F$ : $J \times \mathscr{B}_{\alpha} \rightarrow H_{\alpha+\beta}$ and $G: J \times \mathscr{B}_{\alpha} \rightarrow \mathscr{P}\left(L_{Q}(K, H)\right)$ are, respectively, measurable and multivalued measurable mapping, where $\mathscr{P}\left(L_{\mathrm{Q}}(K, H)\right)$ is the family of all nonempty subsets of $L_{Q}(K, H)$ and $L_{Q}(K, H)$ denotes the space of all $Q$-Hilbert-Schmidt operators from $K$ into $H$. The histories $x_{t}:(-\infty, 0] \rightarrow H_{\alpha}, x_{t}(\theta)=x(t+\theta), \theta \leq 0$, belong to an abstract phase space $\mathscr{B}_{\alpha}$.

The aim of the present work is to investigate the approximate controllability for (3) by using the resolvent operator theory. Because the nonlinear terms involve frequently special derivatives in many practical models and the history variables of the functions $F$ and $G$ are only defined on $C\left([-r, 0] ; H_{1 / 2}\right)$, we cannot discuss the problem on the whole space $H$. We also suppose that there exists a semigroup which generated by $(-A, D(-A))$ is compact analytic on $H$ so that the resolvent $W(t)$ is also analytic. So, we restrict this integrodifferential inclusion in the $H_{\alpha}$ and demonstrate the existence of mild solutions by applying $\|\cdot\|_{\alpha}$ and the fractional power theory and then prove the approximate controllability for (3) in space $H$. In particular, we do not require that the resolvent operator $W(t)$ be compact for $t>0$ which differs greatly from [14].

The whole paper is arranged as follows: in Section 2, we introduce some concepts, hypotheses, and basic results about resolvent operator and approximate controllability. Section 3 is devoted to studying the existence of mild solution of (3) and proving the approximate controllability. The application of our theoretical results is given in Section 4.

\section{Preliminaries}

The complete probability space, denoted by $(\Omega, \mathfrak{F}, P)$, is furnished with a complete family of right continuous increasing sub- $\sigma$-algebras $\left\{\mathfrak{F}_{t}, t \in J\right\}$ satisfying $\mathfrak{F}_{t} \in \mathfrak{F}$. Let the complete orthonormal basis $\left\{e_{n}\right\}_{n \geq 1}$ in $K$ be a bounded sequence of nonnegative real numbers $\lambda_{n}$ such that $Q e_{n}=$ $\lambda_{n} e_{n}(n=1,2,3, \ldots, m)$ and $\omega(t)=\sum_{n=1}^{\infty} \sqrt{\lambda_{n}} \omega_{n}(t) e_{n}$, where $\left\{\omega_{n}(t)\right\}_{n=1}^{\infty}$ are independent one-dimensional standard Wiener processes. We assume that $\mathfrak{F}_{t}=\sigma\{\omega(s): 0 \leq s \leq t\}$ is the sub- $\sigma$-algebra generated by $\omega$ and $\mathfrak{F}_{T}=\mathfrak{F}$. Let $L(K, H)$ be the space of all bounded linear operators from $K$ into $H$ with the usual operator norm $\|\cdot\|$. For $\phi \in L(K, H)$ define

$$
\|\phi\|_{\mathrm{Q}}^{2}=\operatorname{Tr}\left(\phi Q \phi^{*}\right)=\sum_{n=1}^{\infty}\left\|\sqrt{\lambda_{n}} \phi e_{n}\right\|^{2}<\infty .
$$

Then $\phi$ is called a Q-Hilbert-Schmidt operator. Let $\rho(A)$ be the resolvent set of the operator $A$ and $0 \in \rho(A)$; then the fractional power operator $A^{\alpha}, 0<\alpha \leq 1$, is a closed linear operator on $D\left(A^{\alpha}\right)$ in $H$ and the expression

$$
\|x\|_{\alpha}=\left\|A^{\alpha} x\right\|, \quad x \in D\left(A^{\alpha}\right)
$$

defines a norm on $D\left(A^{\alpha}\right)$. Denoting the space $\left(D\left(A^{\alpha}\right),\|\cdot\|_{\alpha}\right)$ by $H_{\alpha}$, then $H_{\alpha}$ is a Banach space for each $0<\alpha \leq 1$. For $0<\beta<\alpha \leq 1$, the imbedding $H_{\alpha} \rightarrow H_{\beta}$ is compact. Meanwhile, $R(\lambda,-A)=(\lambda I+A)^{-1}$, the resolvent operator of $-A$, is compact. Let $L\left(H_{\alpha}, H_{\beta}\right)$ be the space of all bounded linear operators from $H_{\alpha}$ into $H_{\beta}$ with the norm $\|\cdot\|_{\alpha, \beta}$ and $H_{0}=H$. Let $C_{\alpha}:=C\left((-\infty, 0] ; H_{\alpha}\right)$ be the Banach space of continuous functions from $J_{0}$ to $H_{\alpha}$ with the norm

$$
\begin{aligned}
\|x\|_{C_{\alpha}}=\sup _{\theta \in(-\infty, 0]}\left\|A^{\alpha} x(\theta)\right\|, & \\
x & \in C_{\alpha}:=C\left((-\infty, 0] ; H_{\alpha}\right),
\end{aligned}
$$

and the Banach space of continuous functions from $(-\infty, T]$ to $H_{\alpha}$ is defined by $C\left((-\infty, T] ; H_{\alpha}\right)$ with the norm

$$
\|x\|_{C}=\sup _{\theta \in(-\infty, T]}\left\|A^{\alpha} x(\theta)\right\|, \quad x \in C\left((-\infty, T] ; H_{\alpha}\right) .
$$

Keep it simple; let $A^{\alpha} W(t) x=W(t) A^{\alpha} x$, for any $0 \leq \alpha \leq$ $1, x \in D\left(A^{\alpha}\right)$. Let $\mathscr{B}$ be a $\mathfrak{\mho}_{0}$-measurable function from $J_{0}$ 
into $H$ endowed with a seminorm $\|\cdot\|_{\mathscr{B}}$; we assume that $\mathscr{B}$ satisfies the following conditions:

(a) If: $x \in(-\infty, b] \rightarrow H, b>0$, is continuous on $[0, b)$ and $x_{0}$ in $\mathscr{B}$, then for every $t \in[0, b)$ the following conditions hold:

(1) $x_{t}$ is in $\mathscr{B}$;

(2) $\|x(t)\| \leq H_{1}\left\|x_{t}\right\|_{\mathscr{B}}$;

(3) $\left\|x_{t}\right\|_{\mathscr{B}} \leq K(t)\{\sup \|x(s)\|: 0 \leq s \leq t\}+$ $N(t)\left\|x_{0}\right\|_{\mathscr{B}}$, where $H_{1}$ is a positive constant and $K(t)$ and $N(t):[0, \infty) \rightarrow[0, \infty)$ are continuous and independent of $x(\cdot)$.

(b) For the function $x(\cdot)$ in (a), $x_{t}$ is a $\mathscr{B}$-valued function on $[0, b)$.

(c) The space $\mathscr{B}$ is complete.

We define the set of all elements in $\mathscr{B}$ which takes values in space $H_{\alpha}$ by $\mathscr{B}_{\alpha}$. Since $H_{\alpha}$ is still a Banach space, we will assume that the subspace $\mathscr{B}_{\alpha}$ also satisfies the following conditions:

$\left(a^{\prime}\right)$ If: $x \in(-\infty, b] \rightarrow H_{\alpha}, b>0$, is continuous on $[0, b)$ and $x_{0}$ in $\mathscr{B}_{\alpha}$, then for every $t \in[0, b)$ the following conditions hold:

(1) $x_{t}$ is in $\mathscr{B}_{\alpha}$;

(2) $\|x(t)\|_{\alpha} \leq H_{1}\left\|x_{t}\right\|_{\mathscr{B}_{\alpha}}$;

(3) $\left\|x_{t}\right\|_{\mathscr{B}_{\alpha}} \leq K(t)\left\{\sup \left\|A^{\alpha} x(s)\right\|: 0 \leq s \leq\right.$ $t\}+N(t)\left\|x_{0}\right\|_{\mathscr{B}_{\alpha}}$, where $H_{1}$ is a positive constant and $K(t)$ and $N(t):[0, \infty) \rightarrow[0, \infty)$ are continuous and independent of $x(\cdot)$.

$\left(\mathrm{b}^{\prime}\right)$ For the function $x(\cdot)$ in $\left(\mathrm{a}^{\prime}\right), x_{t}$ is a $\mathscr{B}_{\alpha}$-valued function on $[0, b)$.

(c') The space $\mathscr{B}_{\alpha}$ is complete.

Let $L_{2}(\Omega, \mathfrak{F}, P ; H)=L_{2}(\Omega, H)$ be the collection of all strongly measurable, square-integrable $K$-valued stochastic variables with the norm $\|\cdot\|_{L_{2}}=\left(E\|x(\cdot, \omega)\|_{H}^{2}\right)$. The setting $x_{t}=\{x(t+s)(\omega): s \in(-\infty, 0]\}$ is the collection of all $\mathscr{B}_{\alpha}$-valued stochastic process and let $L_{2}\left(\Omega, \mathfrak{F}, P ; H_{\alpha}\right)=$ $L_{2}\left(\Omega, H_{\alpha}\right)$ be the collection of all strongly measurable, square-integrable $K$-valued stochastic variables with the norm $\|\cdot\|_{L_{2}, \alpha}=\left(E\left\|A^{\alpha} x(\cdot, \omega)\right\|_{H}^{2}\right)$.

The space $C\left((-\infty, T], L_{2}^{\mathfrak{F}}\left(\Omega ; \mathscr{B}_{\alpha}\right)\right)$ consists of measurable and $\mathfrak{\mho}_{t}$-adapted processes such that $\phi \in \mathscr{B}_{\alpha}$ and the restriction $x: J \rightarrow L_{2}^{\mathfrak{F}}\left(\Omega ; \mathscr{B}_{\alpha}\right)$ is continuous. The closed subspace of all continuous process $x$ that belongs to $C\left((-\infty, T], L_{2}^{\mathfrak{F}}\left(\Omega ; \mathscr{B}_{\alpha}\right)\right)$ is defined by $Z$ and the seminorm in $Z$ is defined by

$$
\|x\|_{Z}^{2}=\sup _{t \in J}\left\|x_{t}\right\|_{\mathscr{B}_{\alpha}}^{2}
$$

where

$$
\begin{aligned}
\left\|x_{t}\right\|_{\mathscr{B}_{\alpha}} \leq & \bar{N} E\|\phi\|_{\mathscr{B}_{\alpha}} \\
& +\bar{K} \sup \left\{E\left\|A^{\alpha} x(s)\right\|: 0 \leq s \leq T\right\},
\end{aligned}
$$

$\bar{N}=\sup _{t \in J} N(t)$, and $\bar{K}=\sup _{t \in J} K(t)$. It is not hard to prove that $Z$ is a Banach space.
Now, we introduce some facts on multivalues analysis:

(i) If, for each $x \in H$, the set $F(x)$ is a nonempty, closed subset of $H$ and if, for each open set $G$ of $H$ containing $F(x)$, there exists an open neighborhood $c$ of $x$ such that $F(c) \subseteq G$, then $F$ is upper semicontinuous (u.s.c.) on $H$.

(ii) If, for every bounded subset $B \subseteq H, F(B)$ is relatively compact, then $F$ is completely continuous.

(iii) If the multivalued map $F$ is completely continuous with nonempty compact values, then $F$ is upper semicontinuous (u.s.c.) if and only if $F$ has a closed graph (i.e., $x_{n} \rightarrow x, y_{n} \rightarrow y, y_{n} \in F\left(x_{n}\right)$ imply $y \in F(x))$.

For each $x \in L_{2}\left(L_{Q}(K, H)\right)$, define the set of selections of $G$ by

$$
\begin{aligned}
g & \in N_{G, x}=\left\{g \in L_{2}\left(L_{Q}(K, H)\right): g(t)\right. \\
& \left.\in G\left(t, x_{t}\right) \text { for a.e. } t \in J\right\} .
\end{aligned}
$$

Definition 1 (see [19]). A family of bounded linear operators $W(t) \in \mathfrak{Q}(H)$ for $t \in J$ is called resolvent operator for

$$
\begin{aligned}
\frac{d}{d t} x(t) & =-A x(t)+\int_{0}^{t} \gamma(t-s) x(s) d s, \quad t \in J, \\
x(0) & =x_{0} \in H
\end{aligned}
$$

if

(i) $W(0)=I$ and $\|W(t)\| \leq N_{1} e^{\varsigma t}$ for some $N_{1} \geq 0, \varsigma \in R$;

(ii) for all $x \in H, W(t)$ is strongly continuous in $t$ on $J$;

(iii) $W(t) \in \mathfrak{L}(Y)$, for $t \in J$, where $Y$ is the Banach space from $D(-A)$ endowed with the graph norm. Moreover for $x \in Y, W(\cdot) x \in C^{1}(J ; H) \cap C(J ; Y)$ and for $t \geq 0$, the following formula holds:

$$
\begin{aligned}
\frac{d}{d t} W(t) x & =-A W(t) x+\int_{0}^{t} \gamma(t-s) W(s) x d s \\
& =-W(t) A x+\int_{0}^{t} W(t-s) \gamma(s) x d s
\end{aligned}
$$

See from [18-20] that the resolvent operator $W(t)$ for the above linear system exists which is given by $W(0)=I$, and

$$
W(t) x=\frac{1}{2 \pi i} \int_{\Gamma} e^{\lambda t}\left(\lambda I+A-\gamma^{*}(\lambda)\right)^{-1} x d \lambda,
$$

$$
t>0
$$

where $\Gamma$ is contour of the type used to obtain an analytic semigroup. $W(t)$ is also analytic and there exists $N, N_{\alpha}>0$, such that

$$
\begin{aligned}
\|W(t)\| & \leq N, \\
\left\|A^{\alpha} W(t)\right\| & \leq \frac{N_{\alpha}}{t^{\alpha}},
\end{aligned}
$$


Lemma 2 (see [21, Lemma 2.3]). $W(t)$ is continuous for $t>0$ in the uniform operator topology of $\mathbf{Q}(H)$.

Lemma 3 (see [22, Lemma 2.3]). AW( $t$ ) is continuous for $t>$ 0 in the uniform operator topology of $\mathfrak{Q}(H)$.

Definition 4. The multivalued map $G: J * \mathscr{B}_{\alpha} \rightarrow \mathscr{P}(H)$ is said to be $L_{2}$-Carathéodory if

(i) $t \vdash G(t, v)$ is measurable for each $v \in \mathscr{B}_{\alpha}$;

(ii) $v \vdash G(t, v)$ is u.s.c. for almost all $t \in J$;

(iii) for each $q>0$, there exists $h_{q} \in L_{1}\left(J, R_{+}\right)$such that

$$
\begin{aligned}
\|G(t, v)\|^{2}=\sup \left\{E\|g\|^{2},\right. & g \in G(t, v)\} \leq h_{q}(t) \\
& \forall\|v\|_{\mathscr{B}_{\alpha}}^{2} \leq q, \text { for a.e } t \in J .
\end{aligned}
$$

Lemma 5 (see [23]). Let I be a compact interval and $Y$ a Hilbert space. Let $G$ be an $L_{2}$-Carathéodory multivalued map with $N_{G, x} \neq \varnothing$ and let $\Theta$ be a linear continuous mapping from $L_{2}(I, Y)$ to $C(I, Y)$. Then the operator

$$
\begin{aligned}
\Theta \circ N_{G}: C(I, Y) & \longrightarrow \mathscr{P}_{b, c l, c v}(C(I, Y)), \\
x & \longmapsto\left(\Theta \circ N_{G}\right)(x)=\Theta\left(N_{G, x}\right)
\end{aligned}
$$

is a closed graph operator in $C(I, Y) \times C(I, Y)$. Here $\mathscr{P}_{b, c l, c v}(C(I, Y))$ denotes the family of nonempty, bound, close, compact convex subset of $C(I, Y)$.

Definition 6. A $\mathfrak{F}_{t}$-adapted stochastic process $x(t) \in$ $C\left((-\infty, T] ; H_{\alpha}\right)$ is the mild solution of (3) if $x(\cdot)$ is a continuous stochastic process on $[0, T)$, the function $A W(t-$ $s) F\left(s, x_{s}\right)$ is integrable for each $s \in[0, t)$, and $g \in N_{G, x}$ is a selection of $G\left(t, x_{t}\right)$ such that

$$
\begin{aligned}
& x(t) \\
& =\left\{\begin{array}{l}
\phi(t) \in L_{2}\left(\Omega, \mathscr{B}_{\alpha}\right), \\
W(t)(\phi(0)+F(0, \phi))-F\left(t, x_{t}\right)+\int_{0}^{t} A W(t-s) F\left(s, x_{s}\right) d s+\int_{0}^{t} W(t-s) B u(s) d s+\int_{0}^{t} W(t-s) g(s) d \omega(s)-\int_{0}^{t} W(t-s) \int_{0}^{s} \lambda(s-v) F\left(v, x_{v}\right) d v d s, \quad t \in J .
\end{array}\right.
\end{aligned}
$$

Theorem 7 (nonlinear alternative for Kakutani maps). Let $Y$ be a Hilbert space, $C$ a closed convex subset of $Y, \mho$ an open subset of $C$, and $0 \in \mho$. Suppose that $\Phi: \bar{\mho} \rightarrow \mathscr{P}_{c, c v}(C)$ is an upper semicontinuous compact map; here $\mathscr{P}_{c, c v}(C)$ denotes the family of nonempty, compact convex subsets of $C$. Then either

(i) $\Phi$ has a fixed point in $\bar{\mho}$, or

(ii) there are $v \in \partial \mho$ and $\lambda \in(0,1)$ with $v \in \lambda \Phi(v)$.

Definition 8. Equation (3) is said to be approximately controllable on the interval $J$ if $\mathfrak{R}(T, \phi)$ is dense in $H$; that is,

$$
\overline{\mathfrak{R}(T, \phi)}=H,
$$

where $\mathfrak{R}(T, \phi)=\left\{x(T, \phi, u), u(\cdot) \in L_{2}(I ; U)\right\}$.

\section{Approximate Controllability}

We introduce the following operators.

(1) The controllability operator Grammian is as follows:

$$
\Gamma_{T}=\int_{0}^{T} W(T-t) B B^{*} W^{*}(T-t) d t .
$$

(2) The resolvent operator is as follows:

$$
R\left(\lambda, \Gamma_{T}\right)=\left(\lambda I+\Gamma_{T}\right)^{-1},
$$

where $B^{*}$ and $W^{*}(t)$ are the adjoints of the operators $B$ and $W(t)$, respectively. We also assume that the operator $R\left(\lambda, \Gamma_{T}\right)$ satisfies the following hypothesis:

$$
\left(H_{0}\right): \lambda R\left(\lambda, \Gamma_{T}\right) \longrightarrow 0
$$
as $\lambda \longrightarrow 0^{+}$in the strong operator topology.
In this section, we discuss the approximate controllability of (3). Firstly, we compare approximate controllability of the semilinear equation (3) with approximate controllability of the associated linear system. Hypothesis $\left(H_{0}\right)$ is equivalent to the fact that the linear control system corresponding to (3)

$$
\begin{aligned}
x(t) & =\phi(t), \quad t \in J_{0} \\
\frac{d}{d t} x(t) & =-A x(t)+\int_{0}^{t} \gamma(t-s) x(s) d s+B u(t),
\end{aligned}
$$

$t \in J$,

is approximately controllable on $J$. For further details for the linear part of semilinear system, we can look up [24]. Secondly, we demonstrate that, for any given $x^{T} \in H_{\alpha}$ and $\lambda \in(0,1]$, we choose a proper control $u^{\lambda}$ to prove that there exists a mild solution $x^{\lambda}(\cdot, \phi, u) \in C\left((-\infty, T] ; H_{\alpha}\right)$ of (3). At last, we prove that $x^{\lambda}(T) \rightarrow x^{T}$ in $H_{\alpha}$ which implies that (3) is approximately controllable on $J$. Assume $\alpha \in(0,1)$ is fixed:

$\left(H_{1}\right) B$ is a bounded linear operator from $U$ to $H$ and $\|B\| \leq$ M.

$\left(H_{2}\right)$ For each $t \in J$, there exists a constant $\beta \in(0,1)$ with $1 / 2 \leq \alpha+\beta \leq 1$, such that $\gamma(t) \in L\left(H_{\alpha+\beta}, H\right)$. And there exists a number $M_{1}>0$, such that

$$
\|\gamma(t)\|_{\alpha+\beta, 0} \leq M_{1} \quad \text { for } t \in J
$$


$\left(H_{3}\right)$ The function $F: J \times \mathscr{B}_{\alpha} \rightarrow H_{\alpha+\beta}$ is completely continuous. Moreover, there exists a positive, nondecreasing function $f(\cdot) \in L_{1}(R)$ such that

$$
\|F(t, \phi)\|_{\alpha+\beta}^{2} \leq f\left(\|\phi\|_{\mathscr{B}_{\alpha}}^{2}\right) .
$$

$\left(H_{4}\right) G: J \times \mathscr{B}_{\alpha} \rightarrow \mathscr{P}\left(L_{\mathrm{Q}}(K, H)\right)$ is an $L_{2}$-Carathéodory function.

$\left(H_{5}\right)$ There exists a continuous nondecreasing function $\psi$ : $R_{+} \rightarrow(0, \infty), P \in L_{2}\left(J, R_{+}\right)$such that

$$
\begin{aligned}
\|G(t, v)\|_{Q}^{2} & =\sup \left\{\|g\|_{Q}^{2}: g \in G(t, v)\right\} \\
& \leq P(t) \psi\left(\|v\|_{\mathscr{B}_{\alpha}}^{2}\right) ;
\end{aligned}
$$

for almost all $t \in J, v \in \mathscr{B}_{\alpha}$.
For any $x^{T} \in H_{\alpha}$, let $x_{1}=x^{T}$ and $\lambda \in(0,1]$, and we take the control function $u^{\lambda}(t, x)$, simply denoted by $u(t)$, as follows:

$$
\begin{aligned}
& u(t)=B^{*} W^{*}(T-t) R\left(\lambda, \Gamma_{T}\right)\left[x_{1}\right. \\
& -W(T)(\phi(0)+F(0, \phi))+F\left(T, x_{T}\right) \\
& -\int_{0}^{T} A W(T-s) F\left(s, x_{s}\right) d s \\
& -\int_{0}^{T} W(T-s) g(s) d \omega(s) \\
& \left.+\int_{0}^{T} W(T-s) \int_{0}^{s} \gamma(s-v) F\left(v, x_{v}\right) d v d s\right],
\end{aligned}
$$

We define the operator $P^{\lambda}$ on $C\left((-\infty, T] ; H_{\alpha}\right)$ by using this control as follows:

$$
\begin{aligned}
& \left(P^{\lambda} x\right)(t) \\
& =\left\{\begin{array}{l}
\phi(t), \\
W(t) \phi(0)+W(t) F(0, \phi)-F\left(t, x_{t}\right)+\int_{0}^{t} A W(t-s) F\left(s, x_{s}\right) d s+\int_{0}^{t} W(t-s) g(s) d \omega(s)-\int_{0}^{t} W(t-s) \int_{0}^{s} \gamma(s-v) F\left(v, x_{v}\right) d v d s+\int_{0}^{t} W(t-s) B u(s) d s, \quad t \in J .
\end{array}\right.
\end{aligned}
$$

Theorem 9. Let $\phi \in H_{\alpha}$. If the hypotheses $\left(H_{0}\right)-\left(H_{5}\right)$ are satisfied, then, for each $0<\lambda \leq 1$, the operator $P^{\lambda}$ has a fixed point in $C\left[(-\infty, T], H_{\alpha}\right]$ provided that there exists a constant $M_{*}>0$ so that

$$
\begin{aligned}
& \frac{M_{*}-\left[\left(C_{2}+C_{4}\right)\left(T^{2 \beta-1} /(2 \beta-1)\right)+\left(C_{3}+C_{5}\right)\left(T^{1-2 \alpha} /(1-2 \alpha)\right)\right] f\left(M_{*}\right)}{C_{1}+\left(C_{6}+C_{7}\right) \psi\left(\|v\|_{Z}\right)\left(\int_{0}^{T} P^{2}(s) d s\right)^{1 / 2}}>1, \\
& {\left[\left(C_{2}+C_{4}\right) \frac{T^{2 \beta-1}}{2 \beta-1}+\left(C_{3}+C_{5}\right) \frac{T^{1-2 \alpha}}{1-2 \alpha}\right] \frac{f(p)}{p}<1, \text { for any } p>0, }
\end{aligned}
$$

where

$$
\begin{aligned}
C_{1} & =2 \bar{N} E\|\phi\|_{\mathscr{B}_{\alpha}}^{2}+\bar{K}\left\{14 H_{1}^{2} N^{2}\|\phi\|_{\mathscr{B}_{\alpha}}^{2}+14\left\|A^{-\beta}\right\|^{2}\right. \\
& \cdot N^{2} f\left(\|\phi\|_{\mathscr{B}_{\alpha}}^{2}\right)+\frac{98 M^{4} N^{4} T^{2}}{\lambda^{2}}\left[\left\|x_{1}\right\|_{\alpha}^{2}\right. \\
& \left.+H_{1}^{2} N^{2}\|\phi\|_{\mathscr{B}_{\alpha}}^{2}+\left\|A^{-\beta}\right\|^{2} N^{2} f\left(\|\phi\|_{\mathscr{B}_{\alpha}}^{2}\right)\right] \\
& +14\left\|A^{-\beta}\right\|^{2} f\left(\left\|x_{T}\right\|_{\mathscr{B}_{\alpha}}^{2}\right)+\frac{98 M^{4} N^{4} T^{2}}{\lambda^{2}}\left\|A^{-\beta}\right\|^{2} \\
& \left.\cdot f\left(\left\|x_{T}\right\|_{\mathscr{B}_{\alpha}}^{2}\right)\right\},
\end{aligned}
$$

$$
\begin{aligned}
& C_{2}=14 N_{1-\beta}^{2} T, \\
& C_{3}=14 M_{1}^{2} T^{3} N_{\alpha}^{2}, \\
& C_{4}=\frac{98 M^{4} N^{4} T^{2}}{\lambda^{2}} N_{1-\beta}^{2} T, \\
& C_{5}=\frac{98 M^{4} N^{4} T^{2}}{\lambda^{2}} M_{1}^{2} T^{3} N_{\alpha}^{2}, \\
& C_{6}=14 T r Q_{\alpha}^{2} T^{1-4 \alpha} \\
& C_{7}=\frac{98 M^{4} N^{4} T^{2}}{\lambda^{2}} \operatorname{Tr} Q \frac{N_{\alpha}^{2} T^{1-4 \alpha}}{1-4 \alpha} .
\end{aligned}
$$


Proof. Let $\mathscr{B}_{\alpha}^{\prime}$ be the space of all function $x:(-\infty, T] \rightarrow$ $H_{\alpha}$, such that $x_{0} \in \mathscr{B}_{\alpha}^{\prime}$. The restriction $x: J \rightarrow H_{\alpha}$ is continuous and $\|\cdot\|_{T}$ is a seminorm in $\mathscr{B}_{\alpha}^{\prime}$ which is defined by

$$
\|x\|_{T}=\left\|x_{0}\right\|_{\mathscr{B}_{\alpha}}+\sup \left\{\left\|A^{\alpha} x(s)\right\|: 0 \leq s \leq T\right\}
$$

Let $Z_{T}=C\left((-\infty, T], L_{2}\left(\Omega, \mathscr{B}_{\alpha}^{\prime}\right)\right)$. We define the multivalued map $\Phi: Z_{T} \rightarrow \mathscr{P}\left(Z_{T}\right)$ by $\Phi x$ and the set of $P^{\lambda} \in Z_{T}$ such that

$\left(P^{\lambda}\right)(t)$

$= \begin{cases}\phi(t), & t \in J_{0}, \\ W(t) \phi(0)+W(t) F(0, \phi)-F\left(t, x_{t}\right)+\int_{0}^{t} A W(t-s) F\left(s, x_{s}\right) d s+\int_{0}^{t} W(t-s) g(s) d \omega(s)-\int_{0}^{t} W(t-s) \int_{0}^{s} \gamma(s-v) F\left(v, x_{v}\right) d v d s+\int_{0}^{t} W(t-s) B u(s) d s, & t \in J .\end{cases}$

We will prove that the operator $\Phi$ has a fixed point, which then is a solution of (3). For $\phi \in Z$, let $y(\cdot):(-\infty, T] \rightarrow Z_{T}$ be the function defined by

$$
y(t)= \begin{cases}\phi(t), & t \in J_{0} \\ W(t)+\phi(0), & t \in J\end{cases}
$$

Set $x(t)=z(t)+y(t),-\infty<t \leq T$. It is clear that $x$ satisfies (26) if and only if $z(t)$ satisfies $z_{0}=0$ and

$$
\begin{aligned}
z(t)= & W(t) F(0, \phi)-F\left(t, z_{t}+y_{t}\right) \\
& +\int_{0}^{t} A W(t-s) F\left(s, z_{s}+y_{s}\right) d s \\
& -\int_{0}^{t} W(t-s) \int_{0}^{s} \gamma(s-v) F\left(v, z_{v}+y_{v}\right) d v d s \\
& +\int_{0}^{t} W(t-s) g(s) d \omega(s) \\
& +\int_{0}^{t} W(t-s) B u(s) d s, \quad t \in J .
\end{aligned}
$$

Let $\mathscr{B}_{\alpha}^{\prime 0}=\left\{z_{0} \in \mathscr{B}_{\alpha}^{\prime}, z_{0}=0 \in \mathscr{B}_{\alpha}\right\}$. For any $z \in \mathscr{B}_{\alpha}^{\prime 0}$, we have

$$
\begin{aligned}
\|z\|_{T} & =\left\|z_{0}\right\|_{\mathscr{B}_{\alpha}}+\sup \left\{\left\|A^{\alpha} z(s)\right\|: s \in J\right\} \\
& =\sup \left\{\left\|A^{\alpha} z(s)\right\|: s \in J\right\} .
\end{aligned}
$$

So, if $Z_{T}^{0}=C\left(J_{1}, L_{2}\left(\Omega, \mathscr{B}_{\alpha}^{\prime 0}\right)\right)$, then $\left(Z_{T}^{0},\|\cdot\|_{T}\right)$ is a Banach space. For any $q \geq 0$, set

$$
B_{q}=\left\{z \in Z_{T}^{0}:\|z\|_{T}^{2} \leq q\right\} ;
$$

then $B_{q} \subseteq Z_{T}^{0}$ is uniformly bounded, and we have

$$
\begin{aligned}
& \left\|y_{t}\right\|_{\mathscr{B}_{\alpha}}^{2} \\
& \leq\left(\bar{K} \sup \left\{E\left\|A^{\alpha} y(s)\right\|: 0 \leq s \leq T\right\}+\bar{N} E\left(\|\phi\|_{\mathscr{B}_{\alpha}}\right)\right)^{2} \\
& \leq 2 \bar{K}^{2} \sup \{E\|y(s)\| 0 \leq s \leq t\}^{2}+2 \bar{N}^{2} E\|\phi\|_{\mathscr{B}_{\alpha}}^{2} \\
& \leq 2 \bar{K}^{2} N^{2}\|\phi(0)\|^{2}+2 \bar{N}^{2} E\|\phi\|_{\mathscr{B}_{\alpha}}^{2} .
\end{aligned}
$$

For $z \in B(q)$, we can get

$$
\begin{aligned}
& \left\|z_{t}+y_{t}\right\|_{\mathscr{B}_{\alpha}}^{2} \leq 2\left(\left\|z_{t}\right\|_{\mathscr{B}_{\alpha}}^{2}+\left\|y_{t}\right\|_{\mathscr{B}_{\alpha}}^{2}\right) \\
& \quad \leq 4\left(\bar{K}^{2}\left(q+N^{2}\left\|A^{\alpha} \phi(0)\right\|^{2}\right)+\bar{N}^{2} E\|\phi\|_{\mathscr{B}_{\alpha}}^{2}\right) \\
& \quad:=\rho .
\end{aligned}
$$

Let the operator $\widehat{\Phi}: Z_{T}^{0} \rightarrow \mathscr{P}\left(Z_{T}^{0}\right)$ be defined by $\widehat{\Phi}_{z}$, the set of $\widehat{P}^{\lambda} \in Z_{T}^{0}$, such that

$\left(\hat{P}^{\lambda} x\right)(t)$

$= \begin{cases}0, & t \in J_{0}, \\ W(t) F(0, \phi)-F\left(t, x_{t}\right)+\int_{0}^{t} A W(t-s) F\left(s, x_{s}\right) d s+\int_{0}^{t} W(t-s) g(s) d \omega(s)+\int_{0}^{t} W(t-s) B u(s) d s-\int_{0}^{t} W(t-s) \int_{0}^{s} \gamma(s-v) F\left(v, x_{v}\right) d v d s, & x \in J .\end{cases}$ 
We divide the proof into five steps.

Step 1. $\widehat{\Phi} z$ is convex for each $z \in Z_{T}^{0}$.

If $\widehat{P}_{1}^{\lambda}$ and $\widehat{P}_{2}^{\lambda}$ belong to $\widehat{\Phi}_{z}$, then there exists $g_{1}, g_{2} \in N_{G, z}$ such that

$$
\begin{aligned}
\widehat{P}_{i}^{\lambda}(t)= & W(t) F(0, \phi)-F\left(t, x_{t}\right) \\
& +\int_{0}^{t} A W(t-s) F\left(s, x_{s}\right) d s \\
& +\int_{0}^{t} W(t-s) g_{i}(s) d \omega(s) \\
& +\int_{0}^{t} W(t-s) B u(s) d s \\
& -\int_{0}^{t} W(t-s) \int_{0}^{s} \gamma(s-v) F\left(v, x_{v}\right) d v d s, \\
& \quad i=1,2, t \in J .
\end{aligned}
$$

Let $r \in[0,1]$, because the operators $B, B^{*}, W^{*}$ are linear, $t \in J$; we have

$$
\begin{aligned}
& \left(r \widehat{P}_{1}^{\lambda}+(1-r) \widehat{P}_{2}^{\lambda}\right)(t)=W(t) F(0, \phi)-F\left(t, z_{t}+y_{t}\right) \\
& +\int_{0}^{t} A W(t-s) F\left(s, x_{s}\right) d s+\int_{0}^{t} W(t-s) \\
& \cdot\left(r g_{1}(s)+(1-r) g_{2}(s)\right) d \omega(s) \\
& +\int_{0}^{t} W(t-s) B^{*} W^{*}(T-s) R\left(\lambda, \Gamma_{T}\right)\left[x_{1}\right. \\
& -W(T)\left(\phi(0)+F\left(T, x_{T}\right)\right. \\
& \left.-\int_{0}^{T} A W(T-s) F\left(s, z_{s}+y_{s}\right) d s\right) \\
& \left.+\int_{0}^{T} W(T-s) \int_{0}^{s} \gamma(s-v) F\left(v, z_{v}+y_{v}\right) d v\right] d s \\
& -\int_{0}^{T} W(T-s)\left(r g_{1}(s)\right. \\
& \left.+(1-r) g_{2}(s)\right) d \omega(s) .
\end{aligned}
$$

Since $G$ has convex values, $N_{G, z}$ is convex. Then $r \widehat{P}_{1}^{\lambda}+(1-$ $r) \widehat{P}_{2}^{\lambda} \in \widehat{\Phi}_{z}$.

Step 2. $\widehat{\Phi}\left(B_{q}\right)$ is bounded.

The proof can be found in Appendix A.

Step 3. We prove that $\widehat{\Phi}\left(B_{q}\right)$ is equicontinuous.

The proof can be found in Appendix B.

As a consequence of Steps 2 and 3, we can find that $\widehat{\Phi}$ : $Z_{T}^{0} \rightarrow \mathscr{P}\left(Z_{T}^{0}\right)$ is compact multivalued map.

Step 4. $\widehat{\Phi}$ has a closed graph.

The proof can be found in Appendix C.
We will prove that $\widehat{\Phi}$ has a fixed point.

Step 5. We will prove that there exists an open set $\mho \in Z$ and $x \in \partial \mho$, such that $x \notin \lambda \widehat{\Phi} x$, for $\lambda \in(0,1)$.

Let $x \in \lambda \widehat{\Phi}(x)$ for $\lambda \in(0,1)$; then there exists $g \in N_{G, x}$ such that

$$
\begin{aligned}
x(t)= & \lambda W(t)(\phi(0)+F(0, \phi))-\lambda F\left(t, x_{t}\right) \\
& +\lambda \int_{0}^{t} A W(t-s) F\left(s, x_{s}\right) d s \\
& +\lambda \int_{0}^{t} W(t-s) B u(s) d s \\
& -\lambda \int_{0}^{t} W(t-s) \int_{0}^{s} \lambda(s-v) F\left(v, x_{v}\right) d v d s \\
& +\lambda \int_{0}^{t} W(t-s) g(s) d \omega(s) ;
\end{aligned}
$$

by hypotheses $\left(H_{1}\right)-\left(H_{5}\right)$, for each $t \in J$, we have

$$
\begin{aligned}
& E\left(\|x(t)\|_{\alpha}^{2}\right) \leq 7 H_{1}^{2} N^{2}\|\phi\|_{\mathscr{B}_{\alpha}}^{2}+7\left\|A^{-\beta}\right\|^{2} \\
& \cdot N^{2} f\left(\|\phi\|_{\mathscr{B}_{\alpha}}^{2}\right)+\frac{49 M^{4} N^{4} T^{2}}{\lambda^{2}}\left[\left\|x_{1}\right\|_{\alpha}^{2}+H_{1}^{2} N^{2}\|\phi\|_{\mathscr{B}_{\alpha}}^{2}\right. \\
& \left.+\left\|A^{-\beta}\right\|^{2} N^{2} f\left(\|\phi\|_{\mathscr{B}_{\alpha}}^{2}\right)\right]+7\left\|A^{-\beta}\right\|^{2} f\left(\left\|x_{t}\right\|_{\mathscr{B}_{\alpha}}^{2}\right) \\
& +7 N_{1-\beta}^{2} T \int_{0}^{t} \frac{f\left(\left\|x_{s}\right\|_{\mathscr{B}_{\alpha}}^{2}\right)}{(t-s)^{2 \beta-1}} d s \\
& +7 M_{1}^{2} T^{3} N_{\alpha}^{2} \int_{0}^{t} \frac{f\left(\left\|x_{s}\right\|_{\mathscr{B}_{\alpha}}^{2}\right)}{(t-s)^{2 \alpha}} d s \\
& +\frac{49 M^{4} N^{4} T^{2}}{\lambda^{2}}\left[\left\|A^{-\beta}\right\|^{2} f\left(\left\|x_{T}\right\|_{\mathscr{B}_{\alpha}}^{2}\right)\right. \\
& +N_{1-\beta}^{2} T \int_{0}^{T} \frac{f\left(\left\|x_{s}\right\|_{\mathscr{F}_{\alpha}}^{2}\right)}{(t-s)^{2 \beta-1}} d s \\
& \left.+M_{1}^{2} T^{3} N_{\alpha}^{2} \int_{0}^{T} \frac{f\left(\left\|x_{s}\right\|_{\mathscr{B}_{\alpha}}^{2}\right)}{(t-s)^{2 \alpha}} d s\right]+7\left\|A^{\alpha-1}\right\|^{2} \\
& \cdot \operatorname{Tr} Q\left(\int_{0}^{t}\|A W(t-s)\|^{4} d s\right)^{1 / 2} \\
& \cdot\left(\int_{0}^{t} P^{2}(s) \psi^{2}\left(\left\|x_{s}\right\|_{\mathscr{B}_{\alpha}}^{2}\right)\right)^{1 / 2} d s+\left\|A^{\alpha-1}\right\|^{2} \\
& \cdot \frac{49 M^{4} N^{4} T^{2}}{\lambda^{2}} \operatorname{Tr} Q\left(\int_{0}^{T}\|A W(t-s)\|^{4} d s\right)^{1 / 2} \\
& \cdot\left(\int_{0}^{T} P^{2}(s) \psi^{2}\left(\left\|x_{s}\right\|_{\mathscr{B}_{\alpha}}^{2}\right)\right)^{1 / 2} d s .
\end{aligned}
$$


Then, we define the function $v(t)=\sup \left\{\left\|x_{s}\right\|_{\mathscr{B}_{\alpha}}^{2}: 0 \leq s \leq t\right\}$, $t \in J$. We have

$$
\begin{aligned}
v( & \leq 2 \bar{N} E\|\phi\|_{\mathscr{B}_{\alpha}}^{2}+2 \bar{K}\left\{E\|x(t)\|_{\alpha}^{2}\right\} \leq 2 \bar{N} E\|\phi\|_{\mathscr{B}_{\alpha}}^{2} \\
& +2 \bar{K}\left\{7 H_{1}^{2} N^{2}\|\phi\|_{\mathscr{B}_{\alpha}}^{2}+7\left\|A^{-\beta}\right\|^{2} N^{2} f\left(\|\phi\|_{\mathscr{B}_{\alpha}}^{2}\right)\right. \\
& +\frac{49 M^{4} N^{4} T^{2}}{\lambda^{2}}\left[\left\|x_{1}\right\|_{\alpha}^{2}+H_{1}^{2} N^{2}\|\phi\|_{\mathscr{B}_{\alpha}}^{2}\right. \\
& \left.+\left\|A^{-\beta}\right\|^{2} N^{2} f\left(\|\phi\|_{\mathscr{R}_{\alpha}}^{2}\right)\right]+7\left\|A^{-\beta}\right\|^{2} f\left(\left\|x_{t}\right\|_{\mathscr{B}_{\alpha}}^{2}\right) \\
& +7 N_{1-\beta}^{2} T \int_{0}^{t} \frac{f\left(\left\|x_{s}\right\|_{\mathscr{B}_{\alpha}}^{2}\right)}{(t-s)^{2 \beta-1}} d s \\
& +7 M_{1}^{2} T^{3} N_{\alpha}^{2} \int_{0}^{t} \frac{f\left(\left\|x_{s}\right\|_{\mathscr{B}_{\alpha}}^{2}\right)}{(t-s)^{2 \alpha}} d s \\
& +\frac{49 M^{4} N^{4} T^{2}}{\lambda^{2}}\left[\left\|A^{-\beta}\right\|^{2} f\left(\left\|x_{T}\right\|_{\mathscr{B}_{\alpha}}^{2}\right)\right. \\
+ & \left.M_{1}^{2} T^{3} N_{\alpha}^{2} \int_{0}^{T} \frac{f\left(\left\|x_{s}\right\|_{\mathscr{B}_{\alpha}}^{2}\right)}{(t-s)^{2 \alpha}} d s\right]+7\left\|A^{\alpha-1}\right\|^{2} \\
+ & N_{1-\beta}^{2} T \int_{0}^{T} \frac{f\left(\left\|x_{s}\right\|_{\mathscr{B}_{\alpha}}^{2}\right)}{(t-s)^{2 \beta-1}} d s
\end{aligned}
$$

$\cdot \operatorname{Tr} Q\left(\int_{0}^{t}\|A W(t-s)\|^{4} d s\right)^{1 / 2}$

$$
\cdot\left(\int_{0}^{t} P^{2}(s) \psi^{2}\left(\left\|x_{s}\right\|_{\mathscr{B}_{\alpha}}^{2}\right)\right)^{1 / 2} d s+\left\|A^{\alpha-1}\right\|^{2}
$$$$
\cdot \frac{49 M^{4} N^{4} T^{2}}{\lambda^{2}} \operatorname{Tr} Q\left(\int_{0}^{T}\|A W(t-s)\|^{4} d s\right)^{1 / 2}
$$$$
\left.\cdot\left(\int_{0}^{T} P^{2}(s) \psi^{2}\left(\left\|x_{s}\right\|_{\mathscr{B}_{\alpha}}^{2}\right)\right)^{1 / 2} d s\right\} \leq C_{1}
$$$$
+C_{2} \int_{0}^{t} \frac{f\left(\left\|x_{s}\right\|_{\mathscr{B}_{\alpha}}^{2}\right)}{(t-s)^{2-2 \beta}} d s+C_{3} \int_{0}^{t} \frac{f\left(\left\|x_{s}\right\|_{\mathscr{F}_{\alpha}}^{2}\right)}{(t-s)^{2 \alpha}} d s
$$$$
+C_{4} \int_{0}^{T} \frac{f\left(\left\|x_{s}\right\|_{\mathscr{B}_{\alpha}}^{2}\right)}{(t-s)^{2-2 \beta}} d s+C_{5} \int_{0}^{T} \frac{f\left(\left\|x_{s}\right\|_{\mathscr{F}_{\alpha}}^{2}\right)}{(t-s)^{2 \alpha}} d s
$$$$
+C_{6}\left(\int_{0}^{t} P^{2}(s) \psi^{2}\left(\left\|x_{s}\right\|_{\mathscr{B}_{\alpha}}^{2}\right) d s\right)^{1 / 2}
$$$$
+C_{7}\left(\int_{0}^{T} P^{2}(s) \psi^{2}\left(\left\|x_{s}\right\|_{\mathscr{B}_{\alpha}}^{2}\right) d s\right)^{1 / 2}, \quad t \in J
$$

If $t^{*} \in J_{0}$, then $v(t)=\|\phi\|_{\mathscr{B}_{\alpha}}^{2}$ and the previous inequality holds. Consequently,

$$
\begin{gathered}
\|v\|_{Z} \leq C_{1}+\left[\left(C_{2}+C_{4}\right) \frac{T^{2 \beta-1}}{2 \beta-1}+\left(C_{3}+C_{5}\right) \frac{T^{1-2 \alpha}}{1-2 \alpha}\right] f\left(\|v\|_{Z}\right)+\left(C_{6}+C_{7}\right) \psi\left(\|v\|_{Z}\right)\left(\int_{0}^{T} P^{2}(s) d s\right)^{1 / 2} \\
\Longrightarrow \frac{\|v\|_{Z}-\left[\left(C_{2}+C_{4}\right)\left(T^{2 \beta-1} /(2 \beta-1)\right)+\left(C_{3}+C_{5}\right)\left(T^{1-2 \alpha} /(1-2 \alpha)\right)\right] f\left(\|v\|_{Z}\right)}{C_{1}+\left(C_{6}+C_{7}\right) \psi\left(\|v\|_{Z}\right)\left(\int_{0}^{T} P^{2}(s) d s\right)^{1 / 2}} \leq 1 .
\end{gathered}
$$

Then, according to the hypotheses, there exists $M^{*}$ such that $\|x\|_{Z} \neq M^{*}$ and set $\mho=\left\{u \in:\|u\|_{Z}<M^{*}\right\}$. It is clear that there is no $v \in \partial \mho$ for $\lambda \in(0,1)$ such that $v \in \lambda \widehat{\Phi}(v)$. So we deduce that $\widehat{\Phi}$ has a fixed point by Theorem 7 and (3) has a mild solution.

In the end, we prove that (3) is approximately controllable on $J$.

Theorem 10. Assume that the hypotheses $\left(H_{0}\right)-\left(H_{5}\right)$ are satisfied, and the functions $F$ and $G$ are uniformly bounded in the space $H$; then (3) is approximately controllable on $J$.
Proof. Let $x_{1}=x^{T} \in H$, and $x^{\lambda}(\cdot):(-\infty, T] \rightarrow H$ is a mild solution of (3) which is got from Theorem 9. From the definition of $\Gamma_{T}, x^{\lambda}(\cdot)$ satisfies

$$
\begin{gathered}
x^{\lambda}(T)=W(T)(\phi(0)+F(0, \phi))-F\left(T, x_{T}^{\lambda}\right) \\
+\int_{0}^{T} A W(T-s) F\left(s, x_{s}^{\lambda}\right) d s-\int_{0}^{T} W(T-s) \\
\cdot \int_{0}^{s} \gamma(s-v) F\left(v, x_{v}^{\lambda}\right) d v d s+\int_{0}^{T} W(T-s)
\end{gathered}
$$




$$
\begin{aligned}
& g(s) d \omega(s)+\int_{0}^{T} W(T-s) \\
& \cdot B B^{*} W^{*}(T-s) R\left(\lambda, \Gamma_{T}\right)\left\{x_{1}\right. \\
& -\left[W(T)(\phi(0)+F(0, \phi))-F\left(T, x_{T}^{\lambda}\right)\right. \\
& +\int_{0}^{T} A W(T-s) F\left(s, x_{s}^{\lambda}\right) d s \\
& +\int_{0}^{T} W(T-s) g(s) d \omega(s) \\
& \left.\left.-\int_{0}^{T} W(T-s) \int_{0}^{s} \gamma(s-v) F\left(v, x_{v}^{\lambda}\right) d v d s\right]\right\} d s \\
& =W(T)(\phi(0)+F(0, \phi))-F\left(T, x_{T}^{\lambda}\right) \\
& +\int_{0}^{T} A W(T-s) F\left(s, x_{s}^{\lambda}\right) d s-\int_{0}^{T} W(T-s) \\
& \cdot \int_{0}^{s} \gamma(s-v) F\left(v, x_{v}^{\lambda}\right) d v d s+\int_{0}^{T} W(T-s) \\
& \cdot g(s) d \omega(s)+\Gamma_{T} R\left(\lambda, \Gamma_{T}\right)\left\{x_{1}\right. \\
& -\left[W(T)(\phi(0)+F(0, \phi))-F\left(T, x_{T}^{\lambda}\right)\right. \\
& +\int_{0}^{T} A W(T-s) F\left(s, x_{s}^{\lambda}\right) d s \\
& +\int_{0}^{T} W(T-s) g(s) d \omega(s) \\
& \left.\left.-\int_{0}^{T} W(T-s) \int_{0}^{s} \gamma(s-v) F\left(v, x_{v}^{\lambda}\right) d v d s\right]\right\} \\
& =x_{1}+\left(\Gamma_{T} R\left(\lambda, \Gamma_{T}\right)-I\right)\left(x_{1}-R\right) \text {, }
\end{aligned}
$$

where

$$
\begin{aligned}
R= & W(T)(\phi(0)+F(0, \phi))-F\left(T, x_{T}^{\lambda}\right) \\
& +\int_{0}^{T} A W(T-s) F\left(s, x_{s}^{\lambda}\right) d s \\
& -\int_{0}^{T} W(T-s) \int_{0}^{s} \gamma(s-v) F\left(v, x_{v}^{\lambda}\right) d v d s \\
& +\int_{0}^{T} W(T-s) g(s) d \omega(s) .
\end{aligned}
$$

Or we obtain that

$$
\begin{aligned}
E\left\|x^{\lambda}(T)-x_{1}\right\|^{2} & \leq E\left(\left\|\Gamma_{T}\left(R\left(\lambda, \Gamma_{T}\right)-I\right)\right\|\left\|x_{1}-R\right\|\right)^{2} \\
& =E\left\|\lambda R\left(\lambda, \Gamma_{T}\right)\left(x_{1}-R\right)\right\|^{2} .
\end{aligned}
$$

Then, we have

$$
\begin{aligned}
& E\left\|x_{1}-R\right\|^{2} \leq E\left(\| x_{1}-W(T)(\phi(0)+F(0, \phi))\right. \\
& +F\left(T, x_{T}^{\lambda}\right)-\int_{0}^{T} A W(T-s) F\left(s, x_{s}^{\lambda}\right) d s \\
& +\int_{0}^{T} W(T-s) \int_{0}^{s} \gamma(s-v) F\left(v, x_{v}^{\lambda}\right) d v d s \\
& \left.+\int_{0}^{T} W(T-s) g(s) d \omega(s) \|^{2}\right) \leq 6 E\left(\left\|x_{1}\right\|^{2}\right. \\
& +\|W(T)(\phi(0)+F(0, \phi))\|^{2}+\left\|F\left(T, x_{T}^{\lambda}\right)\right\|^{2} \\
& +\left\|\int_{0}^{T} A W(T-s) F\left(s, x_{s}^{\lambda}\right) d s\right\|^{2} \\
& +\left\|\int_{0}^{T} W(T-s) g(s) d \omega(s)\right\|^{2} \\
& \left.+\left\|\int_{0}^{T} W(T-s) \int_{0}^{s} \gamma(s-v) F\left(v, x_{v}^{\lambda}\right) d v d s\right\|^{2}\right) .
\end{aligned}
$$

Now, for $\epsilon>0$ small and $\alpha+\epsilon<1$,

$$
\begin{gathered}
\left\|A^{\alpha+\epsilon} \int_{0}^{T} A W(T-s) F\left(s, x_{s}^{\lambda}\right) d s\right\| \\
\leq \int_{0}^{T} \frac{N_{1+\epsilon-\beta}}{(t-s)^{1+\epsilon-\beta}} d s\left\|F\left(s, x_{s}^{\lambda}\right)\right\|_{\alpha+\beta} .
\end{gathered}
$$

The operator $A^{-(\alpha+\epsilon)}: H \rightarrow H_{\alpha}$ is compactness and the function $F$ is uniformly bounded. So

$$
\begin{aligned}
& \left\{\int_{0}^{T} A W(T-s) F\left(s, x_{s}^{\lambda}\right) d s: \lambda \in(0,1]\right\} \\
& \quad=\left\{A^{-(\alpha+\epsilon)} A^{\alpha+\epsilon} \int_{0}^{T} A W(T-s) F\left(s, x_{s}^{\lambda}\right) d s: \lambda\right. \\
& \quad \in(0,1]\}
\end{aligned}
$$

is relatively compact in $H_{\alpha}$ and $H$. Then, there exists $F_{T}^{1} \in H$ such that

$$
\left\|\int_{0}^{T} A W(T-s) F\left(s, x_{s}^{\lambda}\right) d s\right\|^{2} \longrightarrow F_{T}^{1}, \quad \text { as } \lambda \longrightarrow 0^{+} .
$$

So, we can get $F_{T}^{2}, F_{T}^{3}$, and $F_{T}^{4}$ by the same way, such that

$$
\begin{array}{r}
\left\|F\left(T, x_{T}^{\lambda}\right)\right\|^{2} \rightarrow F_{T}^{2}, \\
\left\|\int_{0}^{T} W(T-s) \int_{0}^{s} \gamma(s-v) F\left(v, x_{v}^{\lambda}\right) d v d s\right\|^{2} \rightarrow F_{T}^{3}, \\
\left\|\int_{0}^{T} W(T-s) g(s) d \omega(s)\right\|^{2} \rightarrow F_{T}^{4},
\end{array}
$$


respectively, as $\lambda \rightarrow 0^{+}$in space $H$. Clearly, $\left\|x_{1}-R\right\|^{2}$ is bounded, and there exists a positive constant $M^{\prime \prime}$ such that $E\left(\left\|x_{1}-R\right\|^{2}\right) \leq M^{\prime \prime}$. According to $\left(H_{0}\right)$, we have

$$
\begin{aligned}
E\left(\left\|x^{\lambda}(T)-x_{1}\right\|^{2}\right) & \leq E\left(\left\|\lambda R\left(\lambda, \Gamma_{T}\right)\right\|^{2}\left\|x_{1}-R\right\|^{2}\right) \\
& \longrightarrow 0 \text { as } \lambda \longrightarrow 0^{+} .
\end{aligned}
$$

So $x^{\lambda}(T) \rightarrow x^{T}$ holds in $H$, and we obtain the approximate controllability of (3).

The proof is finished.

\section{Example}

In this section, we study the following neutral inclusion which arises in the study of heat flow in materials of the so-called retarded type $[25,26]$ as an application to Theorem 10 :

$$
\begin{aligned}
& \frac{\partial}{\partial t}\left[v(t, x)+\int_{-\infty}^{t} a(s-t, z(s, x)) d s\right] \\
& \in \frac{\partial^{2}}{\partial x^{2}} v(t, s)+\int_{0}^{t} b(t-s) \frac{\partial^{2}}{\partial x^{2}} v(s, x) d s \\
& +G(t, v(t-s, x)) d \omega(t)+u(t, x), \\
& v(t, 0)=v(t, \pi)=0, \quad t \geq 0, \\
& v(t, x)=\varphi(t, x), \quad t \in J_{0}, \quad 0 \leq x \leq \pi, t \in J,
\end{aligned}
$$

where $v(t, x)$ represents the temperature of the point $x$ at time $t$, and $a(\cdot, \cdot), G(\cdot, \cdot)$, and $b(\cdot)$ will be described below.

In the space $H=L_{2}[0, \pi], \omega(t)$ stands for cylindrical Wiener process in $H$ defined on a probability space $(\Omega, \mathfrak{F}, P)$. Define the operator $A$ as $A \xi=-\left(\partial^{2} / \partial x^{2}\right) \xi$, where $D(A)=$ $\{v \in H: v, d v / d x$ are absolutely continuous, and $\left.\left(-d^{2} / d x^{2}\right) v \in H, v(0)=v(\pi)\right\}$.

$-A$ generates a strongly continuous semigroup $(S(t))_{t \geq 0}$ which is analytic, compact, and self-adjoint. Moreover, the resolvent operator $R(\lambda,-A)=(\lambda I+A)^{-1}$ is compact when it exists. The complete orthonormal set $\left\{z_{n}\right\}(n=1,2,3, \ldots)$ of eigenvectors of $A$ with $z_{n}=\sqrt{2 / \pi} \sin n x$ also exists. Then, the operator $A^{1 / 2}$ is given by $A^{1 / 2} z=\sum_{n=1}^{\infty} n\left\langle z, z_{n}\right\rangle z_{n}$ on the space $D\left[A^{1 / 2}\right]=\left\{z(\cdot) \in H: \sum_{n=1}^{\infty} n\left\langle\zeta, z_{n}\right\rangle z_{n} \in H\right\}$ and the following properties hold:

(i) If $\zeta \in D(A)$, then $A \zeta=\sum_{n=1}^{\infty} n^{2}\left\langle\zeta, z_{n}\right\rangle z_{n}$.

(ii) If $\zeta \in H$, then $A^{1 / 2} \zeta=\sum_{n=1}^{\infty}(1 / n)\left\langle\zeta, z_{n}\right\rangle z_{n}$.

(iii) The operator $A^{1 / 2}$ is given by $A^{1 / 2} \zeta=\sum_{n=1}^{\infty} n\left\langle\zeta, z_{n}\right\rangle z_{n}$.

Then, rewrite the above system as follows:

$$
\begin{aligned}
& d\left[x(t)+F\left(t, x_{t}\right)\right] \\
& \in\left[-A x(t)+B u(t)+\int_{0}^{t} \gamma(t-s) x(s) d s\right] d t \\
& \quad+G\left(t, x_{t}\right) d \omega(t) .
\end{aligned}
$$

Here we take $\alpha=\beta=1 / 2$ and $\varepsilon<0$ and define the phase space

$$
\mathscr{B}=\left\{\phi \in C\left(J_{0}, H\right): \lim _{\theta \rightarrow-r} e^{\delta \theta} \phi(\theta) \text { exists in } H\right\},
$$

and let $\|\phi\|_{\mathscr{B}}=\sup _{\theta \in J_{0}}\left\{e^{\delta \theta}\|\phi(\theta)\|_{L_{2}}\right\}$. Then $\left(\mathscr{B},\|\phi\|_{\mathscr{B}}\right)$ is a Banach space which satisfies (a)-(c). Meanwhile, the subspace $\mathscr{B}_{1 / 2}$ is defined by

$$
\begin{aligned}
& \mathscr{B}_{1 / 2} \\
& \quad=\left\{\phi \in C\left(J_{0}, H_{1 / 2}\right): \lim _{\theta \rightarrow-r} e^{\delta \theta} \phi(\theta) \text { exists in } H_{1 / 2}\right\},
\end{aligned}
$$

endowed with the norm $\|\phi\|_{\mathscr{B}_{1 / 2}}=\sup _{\theta \in J_{0}}\left\{e^{\delta \theta}\left\|A^{1 / 2} \phi(\theta)\right\|_{L_{2}}\right\}$. Thus for $(t, \phi) \in J \times \mathscr{B}_{1 / 2}$, where $\phi(\theta)(x)=\phi(\theta, x)$, $(\theta, x) \in J_{0} \times[0, \pi]$. Clearly, it satisfies the axioms $\left(\mathrm{a}^{\prime}\right)-\left(\mathrm{c}^{\prime}\right)$. Let $v(t)(x)=v(t, x)$ and define the functions $F: J \times \mathscr{B}_{1 / 2} \rightarrow$ $H, G: J \times \mathscr{B}_{1 / 2} \rightarrow \mathscr{P}\left(L_{\mathrm{Q}}(K, H)\right)$ and $\gamma(t)$ for the infinite delay as follows:

$$
\begin{aligned}
\gamma(t) & =b(t) A \\
F(t, \phi)(x) & =\int_{-\infty}^{0} \int_{0}^{\pi} a(\theta, x, \phi(\theta)(y)) d y d \theta \\
G(t, v)(x) & =\int_{-\infty}^{0} u_{1}(t, x, \theta) G_{1}(v, \theta)(x) d \theta .
\end{aligned}
$$

We assume that the following conditions hold:

(i) $b(t) \in L^{1}\left(R^{+}\right) \bigcap C^{1}\left(R^{+}\right)$with primitive $B(t) \in$ $L_{\text {loc }}^{1}\left(R^{+}\right) . B(t)$ is nonpositive, nondecreasing and $B(0)=1$.

(ii) The function $a(\cdot, \cdot) \in C^{2}$ and there exists a function $a_{1}(\cdot, \cdot) \in L^{1}\left((-\infty, 0] \times R, R^{+}\right)$such that

$$
\begin{aligned}
\left|\frac{\partial^{2}}{\partial x^{2}} a(\theta, z(x))\right| & <a_{1}(\theta)|z(x)| \\
c_{1} & =-\frac{1}{2 \varepsilon} \int_{-\infty}^{0} a_{1}^{2}(\theta) d \theta
\end{aligned}
$$

(iii) The function $u_{1}(t, \xi, \theta) \geq 0$ is continuous in $J \times[0, \pi] \times$ $(-\infty, 0)$,

$$
\begin{aligned}
p(t, x) & =\int_{-\infty}^{0} u_{1}(\theta) d \theta<\infty, \\
Q(t) & =\left(\int_{0}^{\pi} p^{2}(t, x) d x\right)^{1 / 2}<\infty .
\end{aligned}
$$


(iv) The function $G_{1}(\cdot)$ is continuous, $0 \leq G_{1} \leq$ $\psi\left(e^{\theta}\|v(\theta, \cdot)\|_{L_{2}}\right)$, for $(\theta, x) \in J_{0} \times[0, \pi]$, where $\psi(\cdot)$ : $[0, \infty) \rightarrow(0, \infty)$ is continuous and nondecreasing.

Moreover, it is clear that $B=I$ is a bounded and linear operator which satisfies $\left(H_{1}\right)$, and $\gamma(t)$ satisfies condition $\left(\mathrm{H}_{2}\right)$. By condition (ii), we have

$$
\begin{aligned}
& \left\langle F(\phi)(x), z_{n}\right\rangle \\
& =\frac{1}{n}\left\langle\int_{-\infty}^{0} \int_{0}^{\pi} \frac{\partial}{\partial x} a(x, \phi(\theta)(y)) d y d \theta, \bar{z}_{n}(x)\right\rangle \\
& =-\frac{1}{n^{2}}\left\langle\int_{-\infty}^{0} \int_{0}^{\pi} \frac{\partial^{2}}{\partial x^{2}} a(x, \phi(\theta)(y)) d y d \theta, z_{n}(x)\right\rangle,
\end{aligned}
$$

so we get $R(F) \subset D(A)$ and

$$
\|\phi(\theta)\|^{2}=\sum_{n=1}^{\infty}\left\langle\varphi, z_{n}\right\rangle^{2} \leq \sum_{n=1}^{\infty} n^{2}\left\langle\varphi, z_{n}\right\rangle^{2} \leq\|\varphi(\theta)\|_{1 / 2}^{2}
$$

Then, we have

$$
\begin{aligned}
\| F & (\phi)(x) \|_{1}^{2} \\
& \leq \int_{0}^{\pi}\left(\int_{-\infty}^{0}\left|\frac{\partial^{2}}{\partial x^{2}} a(\theta, \phi(\theta)(x))\right| d \theta\right)^{2} d x \\
& \leq \int_{0}^{\pi}\left(\int_{-\infty}^{0} a_{1}(\theta, x)|\phi(\theta)(x)| d y d \theta\right)^{2} d x \\
& \leq \int_{0}^{\pi}\left(\int_{-\infty}^{0} a_{1}(\theta)|\phi(\theta)(x)|_{1 / 2} d \theta\right)^{2} d x \\
& \leq \pi \int_{-\infty}^{0} a_{1}^{2}(\theta) d \theta\left(\int_{0}^{\pi} \int_{-\infty}^{0}|\phi(\theta)(x)|_{1 / 2}^{2} d \theta\right) d x \\
& \leq\left(\pi \int_{-\infty}^{0} a_{1}^{2}(\theta) d \theta\right)\left(\int_{-\infty}^{0} e^{-2 \xi \theta} d \theta\right) \\
& =\left(\sup ^{2}\left\{e^{\xi} \theta\|\phi(\theta)\|_{L_{2}, 1 / 2}\right\}\right)^{2} \\
& \leq\left(e^{2 \xi \theta} \int_{0}^{\pi}|\phi(\theta)(x)|_{1 / 2}^{2} d x\right) \\
& \left.\left(-\frac{1}{2 \xi}\right) \int_{-\infty}^{0} a_{1}^{2}(\theta) d \theta\right)
\end{aligned}
$$

which implies that $\|F(t, \phi)\|^{2} \leq f\left(\|\phi\|_{\mathscr{B}_{1 / 2}}^{2}\right)$ and satisfies the assumption $\left(\mathrm{H}_{3}\right)$. Clearly, according to the definition of $G$, it satisfies the assumption $\left(H_{4}\right)$ and

$$
\begin{aligned}
& \|G(t, \phi)\|_{L_{2}} \\
& =\left[\int_{0}^{\pi}\left(\int_{-\infty}^{0} u_{1}(t, x, \theta) G_{1}(\phi(\theta)(x)) d \theta\right)^{2} d x\right]^{1 / 2} \\
& \leq\left[\int_{0}^{\pi}\left(\int_{-\infty}^{0} u_{1}(t, x, \theta) \psi\left(\|\phi(\theta)(\cdot)\|_{L_{2}}\right) d \theta\right)^{2} d x\right]^{1 / 2} \\
& \leq\left[\int_{0}^{\pi}\left(\int_{-\infty}^{0} u_{1}(t, x, \theta) \psi\left(\|\phi(\theta)\|_{L_{2}, 1 / 2}\right) d \theta\right)^{2} d x\right]^{1 / 2} \\
& \leq\left[\int_{0}^{\pi}\left(\int_{-\infty}^{0} u_{1}(t, x, \theta) \psi\left(e^{\xi \theta}\|\phi(\theta)\|_{L_{2}, 1 / 2}\right) d \theta\right)^{2} d x\right]^{1 / 2} \\
& =\left(\int_{0}^{\pi} p^{2}(t, x) d s\right)^{1 / 2} \psi\left(\|\phi\|_{\mathscr{B}_{1 / 2}}\right)=Q(t) \psi\left(\|\phi\|_{\mathscr{B}_{1 / 2}}\right),
\end{aligned}
$$

which satisfies the assumption $\left(H_{5}\right)$.

To sum up, the conditions stated in Theorem 10 have been satisfied in the above case; therefore the inclusion is approximately controllable.

\section{Conclusion}

In this paper, the approximate controllability of semilinear neutral stochastic integrodifferential inclusions with infinite delay has been studied. A new set of sufficient conditions are formulated for the approximately controllable (3). The family $\lambda(t)$ are closed linear operators and the standard semigroup approaches cannot be used here. The sufficient conditions are established in Hilbert space by applying the theory of analytic resolvent operator, the fractional power theory, and the theorem of nonlinear alternative for Kakutani maps under the assumption that the corresponding linear inclusion is approximately controllable. Moreover, an application is provided to illustrate the obtained theoretical results.

Finally, we have to point out here that, like most related work in the literature of distributed parameter control systems, the definition of controllability for inputs in an infinitely dimensional space is not so practical and there is no indication on how to find the controller to achieve the desired state for the system. We expect some important improvement on these issues to appear in the future work. As the impulsive perturbations and state-dependent delay are very common in many real life phenomena, in future we can study the approximate controllability of integrodifferential systems with impulsive effects and state-dependent delay.

\section{Appendices}

\section{A. The Proof of Step 2}

In fact, we only need to prove that, for each $\widehat{P} \in \widehat{\Phi}$ and $z \in B_{q}$, there exists a positive constant $\mathbb{Q}$ such that $\|\widehat{P}\|_{\alpha}^{2} \leq \mathbf{Q}$. 
If $\widehat{P}^{\lambda}(t) \in \widehat{\Phi}_{z}$, then there exists $g \in N_{G, z}$, such that, for each $t \in J$,

$$
\begin{aligned}
\widehat{P}^{\lambda}(t)= & W(t) F(0, \phi)-F\left(t, x_{t}\right) \\
& +\int_{0}^{t} A W(t-s) F\left(s, x_{s}\right) d s \\
& +\int_{0}^{t} W(t-s) g(s) d \omega(s) \\
& +\int_{0}^{t} W(t-s) B u(s) d s \\
& -\int_{0}^{t} W(t-s) \int_{0}^{s} \gamma(s-v) F\left(v, x_{v}\right) d v d s, \\
& \quad i=1,2, t \in J .
\end{aligned}
$$

From (26), we have

$$
\begin{aligned}
& \|u(s)\|^{2}=\| B^{*} W^{*} R\left(\lambda, \Gamma_{T}\right)\left(x_{1}\right. \\
& -W(T)(\phi(0)+F(0, \phi))+F\left(T, x_{T}\right) \\
& -\int_{0}^{T} A W(T-s) F\left(s, x_{s}\right) d s \\
& -\int_{0}^{T} W(T-s) g(s) d \omega(s) \\
& \left.+\int_{0}^{T} W(T-s) \int_{0}^{s} \gamma(s-v) F\left(v, x_{v}\right) d v d s\right) \|^{2} .
\end{aligned}
$$

According to hypotheses $\left(H_{1}\right)-\left(H_{5}\right)$, for $t \in J$, we get

$$
\begin{gathered}
E\left\|u_{y+z}\right\|^{2} \leq \frac{7 M^{2} N^{2}}{\lambda^{2}} E\left[\left\|x_{1}\right\|^{2}+\|W(T) \phi(0)\|^{2}\right. \\
+\|W(T) F(0, \phi)\|^{2}+\left\|F\left(T, x_{y_{T}+z_{T}}\right)\right\|^{2} \\
+\left\|\int_{0}^{T} A W(T-s) F\left(s, x_{s}\right) d s\right\|^{2}+\| \int_{0}^{T} W(T-s) \\
\cdot g(s) d \omega(s)\left\|^{2}+\right\| \int_{0}^{T} W(T-s) \\
\left.\cdot \int_{0}^{s} \gamma(s-v) F\left(v, x_{y_{v}+z_{v}}\right) d v d s \|^{2}\right] \leq \frac{7 M^{2} N^{2}}{\lambda^{2}} \\
\cdot E\left\{\left\|x_{1}\right\|^{2}+\left\|A^{-\alpha}\right\|^{2} H_{1}^{2} N^{2}\|\phi\|_{\mathscr{B}_{\alpha}}^{2}\right.
\end{gathered}
$$

$$
\begin{aligned}
& +N^{2} \operatorname{Tr} Q\left\|h_{\rho}\right\|_{L_{1}}+\left[N^{2}\left\|A^{-(\alpha+\beta)}\right\|^{2}+\left\|A^{-(\alpha+\beta)}\right\|^{2}\right. \\
& \left.+\frac{N_{1-(\alpha+\beta)}^{2} T^{2(\alpha+\beta)}}{2(\alpha+\beta)-1}+\left\|A^{-\alpha}\right\|^{2} M_{1}^{2} \frac{N_{\alpha}^{2} T^{4-2 \alpha}}{1-2 \alpha}\right] \\
& \cdot f(\rho)\}:=G
\end{aligned}
$$

here $h_{\rho}$ is chosen from Definition 4. Furthermore,

$$
\begin{aligned}
& E\left\|\hat{P}^{\lambda}(t)\right\|_{\alpha}^{2}=E\left[\| W(t) F(0, \phi)-F\left(t, z_{t}+y_{t}\right)\right. \\
& +\int_{0}^{t} A W(t-s) F\left(s, z_{s}+y_{s}\right) d s \\
& +\int_{0}^{t} W(t-s) B u(s) d s \\
& +\int_{0}^{t} W(t-s) g(s) d \omega(s) \\
& \left.-\int_{0}^{t} W(t-s) \int_{0}^{s} \lambda(s-v) F\left(v, z_{v}+y_{v}\right) d v d s \|_{\alpha}^{2}\right] \\
& \leq 6 E\left[N^{2}\|F(0, \phi)\|_{\alpha}^{2}+\left\|A^{-\beta}\right\|^{2} f(\rho)\right. \\
& +\left\|\int_{0}^{t} A^{1-\beta} W(t-s) d s\right\|^{2}(\rho) \\
& +\left\|\int_{0}^{t} A^{\alpha} W(t-s) B u(s) d s\right\|^{2} \\
& +\left\|\int_{0}^{t} A^{\alpha} W(t-s) g(s) d \omega(s)\right\|^{2}+f(\rho) \\
& \left.\cdot\left\|\int_{0}^{t} A^{\alpha} W(t-s) M_{1} T d s\right\|^{2}\right] \\
& \leq 6 E\left[\left(\left\|A^{-\beta}\right\|^{2} N^{2}+\left\|A^{-\beta}\right\|^{2}+\frac{T^{2 \beta} N_{1-\beta}^{2}}{2 \beta-1}\right.\right. \\
& \left.+M_{1}^{2} \frac{T^{4-2 \alpha} N_{\alpha}^{2}}{1-2 \alpha}\right) f(\rho)+M^{2} G \frac{N_{\alpha}^{2}}{1-2 \alpha} T^{2-2 \alpha} \\
& +\left\|A^{\alpha-1}\right\|^{2} \operatorname{Tr} Q \psi(\rho)\left(\int_{0}^{t} P^{2}(s) d s\right)^{1 / 2} \\
& \left.\cdot\left(\int_{0}^{t}\|A W(T-s)\|^{4} d s\right)^{1 / 2}\right]:=\mathbf{\Omega} .
\end{aligned}
$$

Then, we have $\left\|\widehat{P}^{\lambda}\right\|^{2} \leq \mathfrak{Q}$, for each $\widehat{P}^{\lambda} \in \widehat{\Phi}\left(B_{q}\right)$. 


\section{B. The Proof of Step 3}

For each $z \in B_{q}$ and $\widehat{P} \in \widehat{\Phi}_{z}$, let $0<t_{1}<t_{2} \leq T$; there exists $g \in N_{G, z}$ such that

$$
\begin{aligned}
& E\left(\left\|\widehat{P}^{\lambda}\left(t_{2}\right)-\widehat{P}^{\lambda}\left(t_{1}\right)\right\|_{\alpha}\right)^{2}=E\left\{\|\left[W\left(t_{2}\right) F(0, \phi)\right.\right. \\
& -F\left(t_{2}, z_{t_{2}}+y_{t_{2}}\right) \\
& +\int_{0}^{t_{2}} A W\left(t_{2}-s\right) F\left(s, z_{s}+y_{s}\right) d s \\
& +\int_{0}^{t_{2}} W\left(t_{2}-s\right) B u(s) d s \int_{0}^{t_{2}} W\left(t_{2}-s\right) \\
& \cdot \int_{0}^{s} \lambda(s-v) F\left(v, z_{v}+y_{v}\right) d v d s \\
& \left.+\int_{0}^{t_{2}} W\left(t_{2}-s\right) g(s) d \omega(s)\right]-\left[W\left(t_{1}\right) F(0, \phi)\right. \\
& -F\left(t_{1}, z_{t_{1}}+y_{t_{1}}\right) \\
& +\int_{0}^{t_{1}} A W\left(t_{1}-s\right) F\left(s, z_{s}+y_{s}\right) d s \\
& +\int_{0}^{t_{1}} W\left(t_{1}-s\right) B u(s) d s-\int_{0}^{t_{1}} W\left(t_{1}-s\right) \\
& \cdot \int_{0}^{s} \lambda(s-v) F\left(v, z_{v}+y_{v}\right) d v d s \\
& \left.\left.+\int_{0}^{t_{1}} W\left(t_{1}-s\right) g(s) d \omega(s)\right] \|_{\alpha}^{2}\right\} \\
& \leq 10 E\left\{\left\|\left(W\left(t_{2}\right)-W\left(t_{1}\right)\right) F(0, \phi)\right\|_{\alpha}^{2}\right. \\
& +\left\|F\left(t_{2}, z_{t_{2}}+y_{t_{2}}\right)-F\left(t_{1}, z_{t_{1}}+y_{t_{1}}\right)\right\|_{\alpha}^{2} \\
& +\| \int_{0}^{t_{1}} A\left(W\left(t_{2}-s\right)-W\left(t_{1}-s\right)\right) \\
& \cdot F\left(s, z_{s}+y_{s}\right) d s\left\|_{\alpha}^{2}+\right\| \int_{t_{1}}^{t_{2}} A W\left(t_{2}-s\right) \\
& F\left(s, z_{s}+y_{s}\right) d s \|_{\alpha}^{2} \\
& +\| \int_{0}^{t_{1}}\left(W\left(t_{2}-s\right)-W\left(t_{1}-s\right)\right) \\
& \cdot \int_{0}^{s} \lambda(s-v) F\left(v, z_{v}+y_{v}\right) d v d s \|_{\alpha}^{2} \\
& +\| \int_{t_{1}}^{t_{2}} W\left(t_{2}-s\right)
\end{aligned}
$$

$$
\begin{aligned}
& \cdot \int_{0}^{s} \lambda(s-v) F\left(v, z_{v}+y_{v}\right) d v d s \|_{\alpha}^{2} \\
& +\left\|\int_{0}^{t_{1}}\left(W\left(t_{2}-s\right)-W\left(t_{1}-s\right)\right) g(s) d \omega(s)\right\|_{\alpha}^{2} \\
& +\left\|\int_{t_{1}}^{t_{2}} W\left(t_{2}-s\right) g(s) d \omega(s)\right\|_{\alpha}^{2} \\
& +\left\|\int_{0}^{t_{1}}\left(W\left(t_{2}-s\right)-W\left(t_{1}-s\right)\right) B u(s) d s\right\|_{\alpha}^{2} \\
& \left.+\left\|\int_{t_{1}}^{t_{2}} W\left(t_{2}-s\right) B u(s) d s\right\|_{\alpha}^{2}\right\} \leq 10 E\left\{\left\|A^{-\beta}\right\|^{2}\right. \\
& \cdot\left\|W\left(t_{2}\right)-W\left(t_{1}\right)\right\|^{2} f(\rho)+\left\|A^{-\beta}\right\|^{2} \| F\left(t_{2}, z_{t_{2}}\right. \\
& \left.+y_{t_{2}}\right)-F\left(t_{1}, z_{t_{1}}+y_{t_{1}}\right) \|_{\alpha+\beta}^{2}+\left[\left\|A^{-\beta}\right\|^{2} T f(\rho)\right. \\
& \left.+\left\|A^{\alpha-1}\right\|^{2} M_{1}^{2} T^{3}+\left\|A^{\alpha-1}\right\|^{2} M^{2} G T\right] \\
& \cdot \int_{0}^{t_{1}}\left\|A W\left(t_{2}-s\right)-A W\left(t_{1}-s\right)\right\|^{2} d s \\
& +\left\|A^{\alpha-1}\right\|^{2} \operatorname{Tr} Q \psi(\rho)\left(\int_{0}^{t_{1}} P^{2}(s) d s\right)^{1 / 2} \\
& \cdot\left(\int_{0}^{t_{1}}\left\|A W\left(t_{1}-s\right)-A W\left(t_{2}-s\right)\right\|^{4} d s\right)^{1 / 2} \\
& +\left\|A^{\alpha-1}\right\|^{2} \operatorname{Tr} Q \psi(\rho)\left(\int_{t_{1}}^{t_{2}} P^{2}(s) d s\right)^{1 / 2} \\
& \cdot\left(\int_{t_{1}}^{t_{2}}\left\|A W\left(t_{2}-s\right)\right\|^{4} d s\right)^{1 / 2}+\left[f(\rho) M_{1}^{2} T^{2}\right. \\
& \left.+M^{2} G\right] \frac{N_{\alpha}^{2}\left(t_{2}-t_{1}\right)^{2-2 \alpha}}{1-2 \alpha}+f(\rho) \\
& \left.\cdot \frac{N_{1-\beta}^{2}\left(t_{2}-t_{1}\right)^{2 \beta}}{2 \beta-1}\right\} \text {. }
\end{aligned}
$$

It is easy to see that the right-hand side of inequality above tends to 0 as $t_{1} \rightarrow t_{2}$ is independent of $z \in B_{q}$. Since $W(t)$ and $A W(t)$ are continuous in the uniform operator topology on $J$ by Lemmas 2 and 3, thus, the set $\left\{\widehat{\Phi} z: z \in B_{q}\right\}$ is equicontinuous in $Z_{T}^{0}$.

Then, we will demonstrate that $\left\{\left(\widehat{P}^{\lambda} z\right) ; z \in B_{q}\right\}$ is relatively compact in $H_{\alpha}$. In fact, if $t \in J_{0}$, then $\widehat{P}^{\lambda}=0$ is relatively compact. Let $t \in J$ be fixed and $z \in B_{q}$; we have

$$
\widehat{P}^{\lambda}(z)=L_{1}(t)+L_{2}(t)
$$


where $L_{1}(t)=W(t) \phi(0)$ and

$$
\begin{aligned}
& L_{2}(t) \\
& \qquad-\quad F\left(t, y_{t}+z_{t}\right)+\int_{0}^{t} A W(t-s) F\left(s, y_{s}+z_{s}\right) d s \\
& \quad+\int_{0}^{t} W(t-s) B u(s) d s \\
& \quad+\int_{0}^{t} W(t-s) g(s) d \omega(s) \\
& \quad-\int_{0}^{t} W(t-s) \int_{0}^{s} \lambda(s-v) F\left(v, y_{v}+z_{v}\right) d v d s, \\
&
\end{aligned}
$$

Since $t$ is fixed, then $L_{1}(t)$ is a single point in $H_{\alpha}$ and we only need to certify that $L_{2}(t)$ is relatively compact. Observe that, for $0<\alpha<\alpha_{1}<1$,

$$
\begin{aligned}
& \left\|A^{\alpha_{1}} L_{2}(t)\right\|^{2} \leq 5\left[\left\|A^{\alpha_{1}} W(t) F\left(t, y_{t}+z_{t}\right)\right\|^{2}\right. \\
& +\left\|\int_{0}^{t} A^{1+\alpha_{1}} W(t-s) F\left(s, y_{s}+z_{s}\right) d s\right\|^{2} \\
& +\left\|\int_{0}^{t} A^{\alpha_{1}} W(t-s) B u(s) d s\right\|^{2} \\
& +\left\|\int_{0}^{t} A^{\alpha_{1}} W(t-s) g(s) d \omega(s)\right\|^{2} \\
& +\| \int_{0}^{t} A^{\alpha_{1}} W(t-s) \\
& \left.\cdot \int_{0}^{s} \gamma(s-v) F\left(v, y_{v}+z_{v}\right) d v d s \|^{2}\right] \\
& \leq 5\left[\left(\left\|A^{-\beta_{1}}\right\|^{2} N^{2}+\frac{N_{1-\beta_{1}}^{2} T^{2 \beta_{1}}}{2 \beta_{1}-1}+M_{1}^{2}\right.\right. \\
& \left.\cdot \frac{N_{\alpha_{1}}^{2} T^{4-2 \alpha_{1}}}{1-2 \alpha_{1}}\right) f(\rho)+M^{2} G \frac{N_{\alpha_{1}}^{2} T^{2-2 \alpha_{1}}}{1-2 \alpha_{1}} \\
& +\left\|A^{\alpha_{1}-1}\right\|^{2} \operatorname{Tr} Q \psi(\rho)\left(\int_{0}^{T} P^{2}(s) d s\right)^{1 / 2} \\
& \left.\cdot\left(\int_{0}^{T}\|A W(T-s)\|^{4} d s\right)^{1 / 2}\right]<+\infty
\end{aligned}
$$

where $\alpha+\beta=\alpha_{1}+\beta_{1}$. It implies that $L_{2}(t)$ is bounded in $H$. Since the operator $A^{-\alpha_{1}}: H \rightarrow H_{\alpha_{1}}$ and the imbedding $H_{\alpha_{1}} \rightarrow H_{\alpha}$ is compact, we infer that $\left(\widehat{P}^{\lambda} z\right)(t)$ is relatively compact in $H_{\alpha}$. Hence, $\left\{(\widehat{\Phi} z)(t): z \in B_{q}\right\}$ is also relatively compact in $H_{\alpha}$.

\section{The Proof of Step 4}

Let $z^{n} \rightarrow z^{*}, \widehat{P}_{n} \rightarrow, \widehat{P}_{z^{n}}, \widehat{P}_{n} \rightarrow \widehat{P}_{*}$; we will prove that $\widehat{P}_{*} \rightarrow$ $\widehat{\Phi}(*)$. Let $g_{n} \in N_{G, z^{n}}$; we have

$$
\begin{aligned}
\widehat{P}_{n}(t) & \\
= & W(t) F(0, \phi)-F\left(t, z_{s}^{n}+y_{s}\right) \\
& +\int_{0}^{t} A W(t-s) F\left(s, z_{s}^{n}+y_{s}\right) d s \\
& -\int_{0}^{t} W(t-s) \int_{0}^{s} \gamma(s-v) F\left(v, z_{v}^{n}+y_{v}\right) d v d s \\
& +\int_{0}^{t} W(t-s) B u_{z^{n}}(s) d s \\
& +\int_{0}^{t} W(t-s) g_{n}(s) d \omega(s),
\end{aligned}
$$

where $u_{z^{n}}(t)$ is obtained from (26) by replacing $x_{t}=y_{t}+z_{t}^{n}$. We will certify that there exists $g_{*} \in N_{G, z^{*}}$ such that $\widehat{P}_{*}(t)$ and respective $u_{z^{*}}(t)$ holds. Let

$$
\begin{aligned}
& \bar{u}_{z}^{T}(t)=B^{*} W^{*}(T-t) R\left(\lambda, \Gamma_{T}\right)\left[x_{1}\right. \\
& -W(T)(\phi(0)+F(0, \phi))+F\left(T, y_{T}+z_{T}\right) \\
& \quad-\int_{0}^{T} A W(T-s) F\left(s, y_{s}+z_{s}\right) d s \\
& \left.\quad+\int_{0}^{T} W(T-s) \int_{0}^{s} \gamma(r-v) F\left(v, y_{s}+z_{s}\right) d v d s\right] .
\end{aligned}
$$

Since $A, W(t)$, and $F\left(t, x_{t}\right)$ are continuous, then $\bar{u}_{z^{n}}(t) \rightarrow$ $u_{z^{*}}(t)$ for $t \in J$. We have

$$
\begin{aligned}
& \|\left(\widehat{P}_{n}(t)-W(t) F(0, \phi)+F\left(t, z_{t}^{n}+y_{t}\right)\right. \\
& -\int_{0}^{t} A W(t-s) F\left(s, z_{s}^{n}+y_{s}\right) d s \\
& +\int_{0}^{t} W(t-s) \int_{0}^{s} \gamma(s-v) F\left(v, z_{v}^{n}+y_{v}\right) d v d s \\
& \left.-\int_{0}^{t} W(t-s) B \bar{u}_{z^{n}}(s) d s\right)-\left(\widehat{P}_{*}(t)\right. \\
& -W(t) F(0, \phi)+F\left(t, z_{s}^{*}+y_{s}\right) \\
& -\int_{0}^{t} A W(t-s) F\left(s, z_{s}^{*}+y_{s}\right) d s \\
& +\int_{0}^{t} W(t-s) \int_{0}^{s} \gamma(s-v) F\left(v, z_{v}^{*}+y_{v}\right) d v d s \\
& \left.\quad-\int_{0}^{t} W(t-s) B \bar{u}_{z^{*}}(s) d s\right) \|^{2} \longrightarrow 0,
\end{aligned}
$$


Consider the linear continuous operator:

$$
\begin{aligned}
\Theta & : L_{2}^{\mathfrak{F}}\left(L_{Q}(K, H)\right) \longrightarrow Z, \\
g & \longmapsto(\Theta g)(t)=\int_{0}^{t} W(t-s)\{g(s) d \omega(s) \\
& -B B^{*} W^{*} R\left(\lambda, \Gamma_{T}\right) \\
& \left.\cdot\left[\int_{0}^{T} W(T-v) g(v) d \omega(v)\right](s) d s\right\} .
\end{aligned}
$$

Obviously, $\Theta$ is linear and continuous; $\Theta \circ N_{G}$ is a closed graph operator from Lemma 5. Moreover,

$$
\begin{aligned}
& \left(\widehat{P}_{n}-W(t) F(0, \phi)+F\left(t, z_{t}^{n}+y_{t}\right)\right. \\
& \quad-\int_{0}^{t} A W(t-s) F\left(s, z_{s}^{n}+y_{s}\right) d s \\
& \quad+\int_{0}^{t} W(t-s) \int_{0}^{s} \gamma(s-v) F\left(v, z_{v}^{n}+y_{v}\right) d v d s \\
& \left.-\int_{0}^{t} W(t-s) B \bar{u}_{z^{n}}(s) d s\right) \in \Theta\left(N_{G, z^{n}}\right) .
\end{aligned}
$$

Since $z^{n} \rightarrow z^{*}$, it follows from Lemma 5 that

$$
\begin{gathered}
\widehat{P}_{*}-W(t) F(0, \phi)+F\left(t, z_{t}^{*}+y_{t}\right)-\int_{0}^{t} A W(t-s) \\
\cdot F\left(s, z_{s}^{*}+y_{s}\right) d s+\int_{0}^{t} W(t-s) \int_{0}^{s} \gamma(s-v) \\
\cdot F\left(v, z_{v}^{*}+y_{v}\right) d v d s-\int_{0}^{t} W(t-s) \\
\cdot B \bar{u}_{z^{*}}(s) d s=\int_{0}^{t} W(t-s)\left\{g_{*}(s) d \omega(s)\right. \\
-B B^{*} W^{*} R\left(\lambda, \Gamma_{T}\right)\left[\int_{0}^{T} W(T-v) g_{*}(v) d \omega(v)\right] \\
\cdot(s) d s\},
\end{gathered}
$$

for some $g_{*} \in N_{G, z^{*}}$. Therefore $\widehat{\Phi}$ is a completely continuous multivalued map, u.s.c. with convex closed values.

\section{Conflict of Interests}

The authors declare that there is no conflict of interests regarding the publication of this paper.

\section{Acknowledgment}

This paper is supported by NNSF of China (no. 11371087).

\section{References}

[1] K. Naito, "Controllability of semilinear control systems dominated by the linear part," SIAM Journal on Control and Optimization, vol. 25, no. 3, pp. 715-722, 1987.

[2] R. Sakthivel, Q. H. Choi, and S. M. Anthoni, "Controllability result for nonlinear evolution integro-differential systems," Applied Mathematics Letters, vol. 17, no. 9, pp. 1015-1023, 2004.

[3] S. Sh. Alaviani, "Controllability of a class of nonlinear neutral time-delay systems," Applied Mathematics and Computation, vol. 232, pp. 1235-1241, 2014.

[4] Y. K. Chang, J. J. Nieto, and W. S. Li, "Controllability of semilinear differential systems with nonlocal initial conditions in Banach spaces," Journal of Optimization Theory and Applications, vol. 142, no. 2, pp. 267-273, 2009.

[5] K. Balachandran and J. Y. Park, "Controllability of fractional integro differential systems in Banach spaces," Nonlinear Analysis. Hybrid Systems, vol. 3, no. 4, pp. 363-367, 2009.

[6] C. Ravichandran and D. Baleanu, "On the controllability of fractional functional integro-differential systems with an infinite delay in Banach spaces," Advances in Difference Equations, vol. 2013, article 291, 13 pages, 2013.

[7] V. Vijayakumar, A. Selvakumar, and R. Murugesu, "Controllability for a class of fractional neutral integro-differential equations with unbounded delay," Applied Mathematics and Computation, vol. 232, pp. 303-312, 2014.

[8] J. Liang and H. Yang, "Controllability of fractional integrodifferential evolution equations with nonlocal conditions," Applied Mathematics and Computation, vol. 254, pp. 20-29, 2015.

[9] P. Balasubramaniam and J. P. Dauer, "Controllability of semilinear stochastic delay evolution equations in Hilbert spaces," International Journal of Mathematics and Mathematical Sciences, vol. 31, no. 3, pp. 157-166, 2002.

[10] J. P. Dauer and N. I. Mahmudov, "Controllability of stochastic semilinear functional differential equations in Hilbert spaces," Journal of Mathematical Analysis and Applications, vol. 290, no. 2, pp. 373-394, 2004.

[11] K. Balachandran, J.-H. Kim, and S. Karthikeyan, "Controllability of semilinear stochastic integrodifferential systems," Kybernetika, vol. 43, no. 1, pp. 31-44, 2007.

[12] R. Subalakshmi, K. Balachandran, and J. Y. Park, "Controllability of semilinear stochastic functional integrodifferential systems in Hilbert spaces," Nonlinear Analysis-Hybrid Systems, vol. 3, no. 1, pp. 39-50, 2009.

[13] L. Shen, J. Shi, and J. Sun, "Complete controllability of impulsive stochastic integro-differential systems," Automatica, vol. 46, no. 6, pp. 1068-1073, 2010.

[14] P. Balasubramaniam and S. K. Ntouyas, "Controllability for neutral stochastic functional differential inclusions with infinite delay in abstract space," Journal of Mathematical Analysis and Applications, vol. 324, no. 1, pp. 161-176, 2006.

[15] N. I. Mahmudov, "Approximate controllability of evolution systems with nonlocal conditions," Nonlinear Analysis-Theory, Methods \& Applications, vol. 68, no. 3, pp. 536-546, 2008.

[16] S. Ji, "Approximate controllability of semilinear nonlocal fractional differential systems via an approximating method," Applied Mathematics and Computation, vol. 236, pp. 43-53, 2014. 
[17] U. Arora and N. Sukavanam, "Approximate controllability of second order semilinear stochastic system with nonlocal conditions," Applied Mathematics and Computation, vol. 258, pp. 111-119, 2015.

[18] F. Z. Mokkedem and X. Fu, "Approximate controllability of semi-linear neutral integro-differential systems with finite delay," Applied Mathematics and Computation, vol. 242, pp. 202215, 2014.

[19] K. Ezzinbi, H. Toure, and I. Zabsonre, "Local existence and regularity of solutions for some partial functional integrodifferential equations with infinite delay in Banach spaces," Nonlinear Analysis: Theory, Methods \& Applications, vol. 70, no. 9, pp. 3378-3389, 2009.

[20] R. C. Grimmer and A. J. Pritchard, "Analytic resolvent operators for integral equations in Banach space," Journal of Differential Equations, vol. 50, no. 2, pp. 234-259, 1983.

[21] X. Fu and R. Huang, "Existence of solutions for neutral integrodifferential equations with state-dependent delay," Applied Mathematics and Computation, vol. 224, pp. 743-759, 2013.

[22] X. Fu, Y. Gao, and Y. Zhang, "Existence of solutions for neutral integrodifferential equations with nonlocal conditions," Taiwanese Journal of Mathematics, vol. 16, no. 5, pp. 1879-1909, 2012.

[23] A. Lasota and Z. Opial, "An application of the Kakutani-KyFan theorem in the theory of ordinary differential equations," Bulletin de l'Académie Polonaise des Sciences, Série des Sciences Mathématiques, Astronomiques et Physiques, vol. 13, pp. 781-786, 1965.

[24] N. I. Mahmudov and S. Zorlu, "Controllability of non-linear stochastic systems," International Journal of Control, vol. 76, no. 2, pp. 95-104, 2003.

[25] A. Lunardi, "On the linear heat equation with fading memory," SIAM Journal on Mathematical Analysis, vol. 21, no. 5, pp. 12131224, 1990.

[26] J. W. Nunziato, "On heat conduction in materials with memory," Quarterly of Applied Mathematics, vol. 29, pp. 187-304, 1971. 


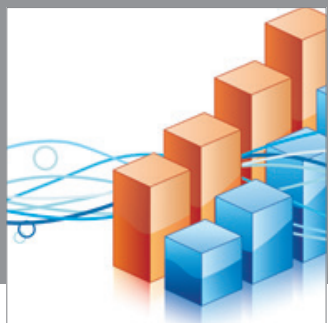

Advances in

Operations Research

mansans

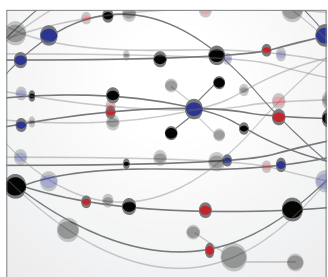

The Scientific World Journal
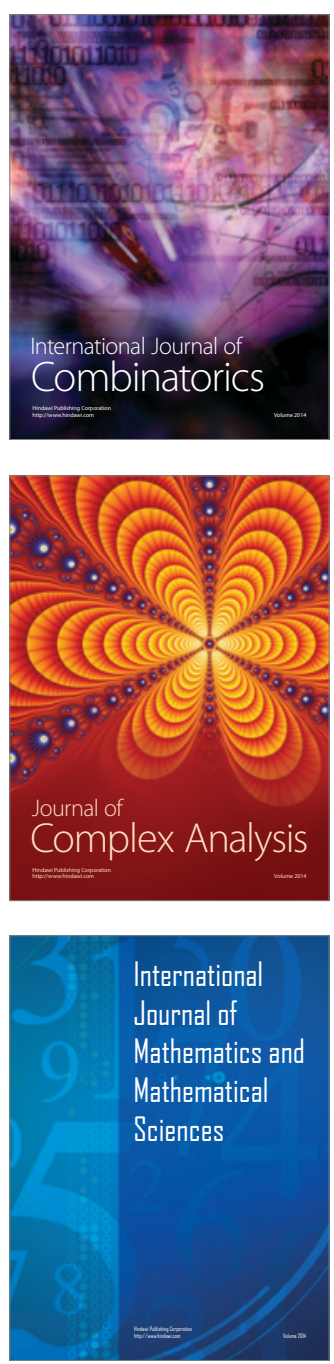
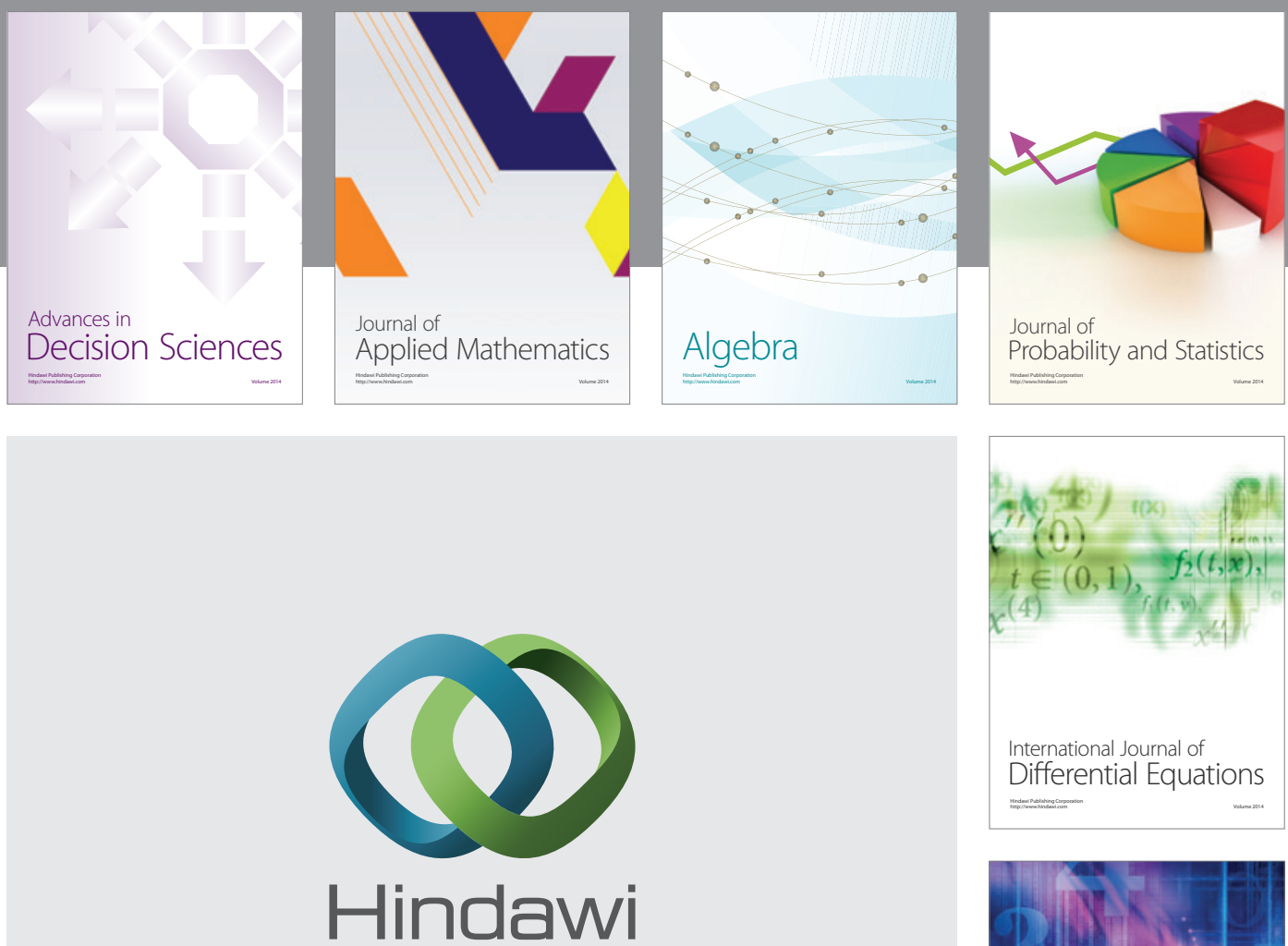

Submit your manuscripts at http://www.hindawi.com
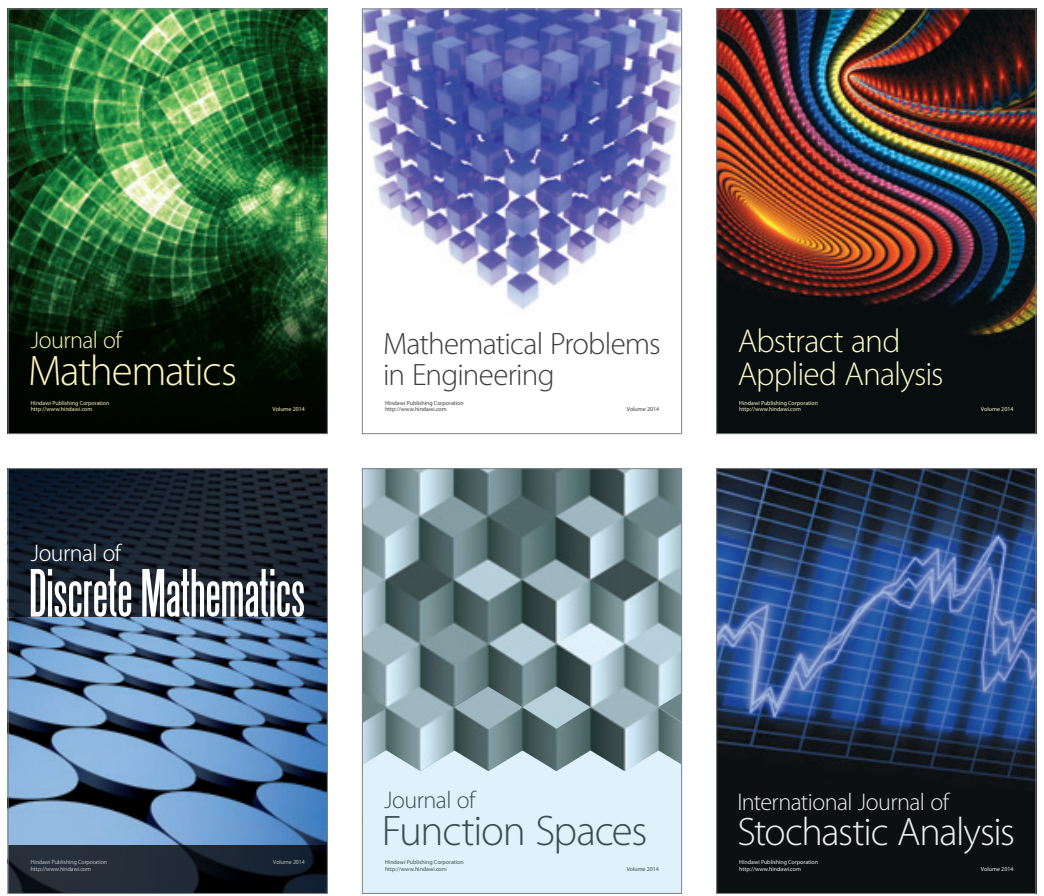

Journal of

Function Spaces

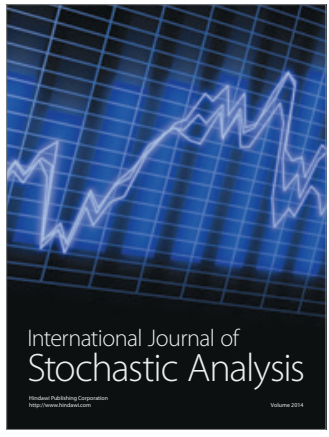

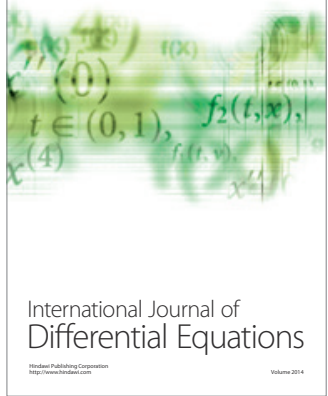
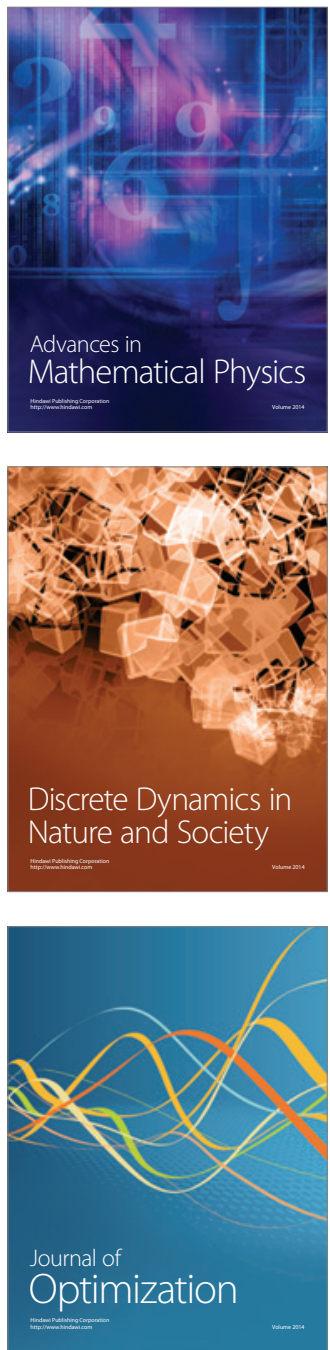